\title{
Coupling an LES approach and a soot sectional model for the study of sooting turbulent non-premixed flames
}

\author{
Pedro RODRIGUES ${ }^{\mathrm{a}, *}$, Benedetta FRANZELLI ${ }^{\mathrm{a}}$, Ronan VICQUELIN ${ }^{\mathrm{a}}$, \\ Olivier GICQUEL ${ }^{\mathrm{a}}$, Nasser DARABIHA ${ }^{\mathrm{a}}$ \\ ${ }^{a}$ Laboratoire EM2C, CNRS, CentraleSupélec, Université Paris-Saclay, \\ Grande Voie des Vignes \\ 92295 Châtenay-Malabry Cedex, France
}

\begin{abstract}
Due to their negative impacts on environment and human health, future regulations on soot emissions are expected to become stricter, in particular by controlling the size of the emitted particles. Therefore, the development of precise and sophisticated models describing the soot production, such as sectional methods, is an urgent scientific and industrial challenge. In this context, the first objective of this work is to use for the first time a sectional model to perform an LES of a sooting turbulent flames in order to demonstrate its capacities. For this, the whole LES formalism for this approach is developed. It includes state-of-art models for the description of the gaseous phase and an extension of a soot subgrid intermittency model to the sectional approach, originally proposed for the hybrid method of moments. Then, the LES is used to analyze a turbulent non-premixed ethylene-air jet diffusion flame and results are validated by available experimental data. The quality of results for the gaseous phase is satisfactory and results for the solid phase show a reasonable agreement with the experimental results in terms of localization, intermittency and soot volume fraction magnitude. Once the coupled LES-sectional approach validated, having access to the full information on the spatial and temporal evolution of the soot Particle Size Distribution (PSD), the second objective of this work is to provide
\end{abstract}

\footnotetext{
${ }^{*}$ Corresponding author:

Email address: pedro.rodrigues@centralesupelec.fr (Pedro RODRIGUES)
}

Preprint submitted to Combustion and Flame

February 27, 2018 
a new fundamental insight on soot production in turbulent non-premixed flames. First, it is observed that a one-peak and a two-peak PSD shapes are observed at the bottom and downstream of the flame, respectively. Second, high fluctuations of the PSD distribution is observed all along the flame. In particular, a time bimodal behavior is observed with the presence of a zone with regular transitions between one- and two-peak PSD shapes. By analyzing soot particles Lagrangian paths, these high fluctuations are shown to be linked with the wide range of history paths of soot particles, which are mainly driven by turbulence. Keywords:

Soot, Sectional model, Particle size distribution, Large Eddy Simulation, Turbulent non-premixed flame

\section{Introduction}

Soot particles result from an incomplete combustion of hydrocarbon fuels and are generally undesirable due to their harmful impacts on both environment [1] and human health [2].

The prediction of soot emission is extremely challenging due to its complex nature, characterized by a strong coupling between flow parameters, flame characteristics and soot properties. This is even more difficult when studying soot production in turbulent flames, where the chemical scales underlying soot production compete with the turbulence scales [3, 4, 5].

Therefore, the numerical prediction of soot requires adequate and precise models for the characterization of the turbulent behaviour of the flame as well of the different phenomena involved in soot production. Different strategies have been proposed in literature as a compromise between accuracy and computational cost. On the one side, Direct Numerical Simulations (DNS), providing a full description of all the temporal and spatial scales, and Large Eddy Simulations (LES), resolving only the most energetic scales, have been used to investigate turbulent soot production in academic configurations $[5,6,7]$ or more realistic flames $[8,9,10,11,12]$, respectively. However, due to their 
high computational cost, these simulations rely on simplified description for the soot evolution, i.e. semi-empirical models $[13,14]$ or methods of moments $[15,16,17,18]$, which usually do not provide access to the soot particle size distribution (PSD). Therefore, these approaches allow an adequate description of the spatial and temporal evolution of the flow and the flame, but not of the soot PSD. Nevertheless, method of moments can provide an accurate description of soot fractality at a low cost, by using bi-variate moments of the soot PSD in particles surface and volume spaces $[16,23,21]$. On the other side, due to their high computational cost, the use of sectional methods, a discretized representation of the soot particle size distribution in the particles volume space, have been limited to Reynolds Averages Navier Stokes (RANS) computations [26, 27, 28]. This RANS-sectional approach provides access to more details in soot particle size distribution spatial evolution while loosing information on the flow and the flame, for which only ensemble-average statistics are available.

In this work, we propose to exploit the whole potential of both strategies, by combining an LES approach with a sectional model for the prediction of soot particles evolution, in order to access new information about soot particles dynamics in turbulent flames through the study of their particle size distributions. To our knowledge, this approach has never been tackled until now.

In this context, the objective of this paper is twofold. First, the feasibility and validity of LES approach based on a sectional model are demonstrated for sooting turbulent non-premixed flames. For this, the soot sectional model is reminded in Sec. 2. Then, the LES formalism is introduced in Sec. 3, by presenting the models for all the unclosed terms of the filtered equations for the solid phase description. In particular, the soot intermittency subgrid model developed in [29] for the hybrid method of moments is extended to the soot sectional model. The model is then applied in Sec. 4 to the simulation of an ethylene/air jet diffusion flame. Temperature and species radial profiles are compared to experiments. Concerning soot particles evolution, axial and radial profiles of mean and root mean square (RMS) of soot volume fraction are compared to experiments. 
Once the LES approach validated and having access to the full information on soot production phenomena, the second objective of this work is to investigate soot production in turbulent flames. Soot formation is then analyzed in Sec. 5 through the study of the different source terms involved in soot production. The major contributors of soot production are then identified. Thanks to the coupled LES-sectional approach, information on the spatial and temporal evolution of the PSD are numerically accessible for the first time, whereas only evolutions about the moments of soot PSD were previously analyzed thanks to the method of moments $[25,22]$. In the current study, high fluctuations between one-peak and two-peak PSD shapes are observed along the flame and soot dynamics are discussed in details, through the study of several soot particles Lagrangian paths.

Finally, an interpretation of the usual time soot intermittency index is proposed in Sec. 6 based on the full temporal data obtained for the particle size distribution. The results for the corresponding index is then compared with other indexes based on other variables representative of soot particles presence and the obtained differences between them are discussed.

\section{Soot sectional model}

The soot sectional model is briefly presented here in order to ease its development in the LES formalism. More details can be found in [28, 30, 31, 33] and in AppendixA. The quality of this sectional model on laminar flames is discussed in the supplementary material of [33].

In the soot sectional approach, the soot particles distribution is discretized in $N_{\text {sect }}$ sections. Each section $i$ represents particles with a volume between $v_{i}^{\min }$ and $v_{i}^{\max }$, for which the soot mass fraction $Y_{s, i}$ is given by the following transport equation:

$$
\frac{\partial \rho Y_{s, i}}{\partial t}+\nabla \cdot\left(\rho\left(\mathbf{u}+\mathbf{v}_{\mathbf{T}}\right) Y_{s, i}\right)=\rho_{s} \dot{Q}_{s, i}
$$


where $\rho$ is the gas phase density, $\mathbf{u}$ is the gas velocity, $\mathbf{v}_{\mathbf{T}}=-C_{\mathrm{th}} \frac{\nu}{T} \nabla T$ [34] is the thermophoretic velocity (with $C_{\mathrm{th}}=0.554$ ), and $\rho_{s}$ is the constant soot density (chosen equal to $\rho_{s}=1860 \mathrm{~kg} / \mathrm{m}^{3}$ ). $\dot{Q}_{s, i}=\rho \dot{q}_{s, i}$ is the production rate (in $\mathrm{s}^{-1}$ ) of the soot volume fraction for the $i^{\text {th }}$ section. Diffusion of soot particles is here neglected since soot particles are characterized by high Schmidt numbers [4].

The production rate $\dot{q}_{s, i}\left(\mathrm{in} \mathrm{m}^{3} \cdot \mathrm{kg}^{-1} \cdot \mathrm{s}^{-1}\right)$ of the soot volume fraction for the $i^{\text {th }}$ section accounts for $[16,23,33]$ :

- nucleation (subscript nu), considered as the coalescence of two dimers,

- condensation (subscript cond), considered as the coalescence of a dimer at a soot particle surface,

- surface growth (subscript sg) and oxidation (subscript ox ), describing the surface reactivity of soot particles,

- coagulation (subscript coag), corresponding to the collision of two solid particles resulting in a bigger soot particle.

It can then be expressed as:

$$
\dot{q}_{s, i}=\dot{q}_{\mathrm{nu}, i}+\dot{q}_{\mathrm{cond}, i}+\dot{q}_{\mathrm{sg}, i}+\dot{q}_{\mathrm{ox}, i}+\dot{q}_{\mathrm{coag}, i} .
$$

The different soot section source terms for nucleation, condensation, surface growth, oxidation, and coagulation are gathered in AppendixA. It is convenient to rewrite all the source terms as a product of two contributions, in order to highlight their dependence on the gaseous and solid characteristics:

$$
\begin{aligned}
& \dot{q}_{\mathrm{nu}, i}=\dot{q}_{\mathrm{nu}, i}^{\mathrm{gas}} \dot{q}_{\mathrm{nu}, i}^{\mathrm{solid}} \\
& \dot{q}_{\mathrm{cond}, i}=\dot{q}_{\mathrm{cond}, i}^{\mathrm{gas}} \dot{q}_{\mathrm{cond}, i}^{\mathrm{solid}} \\
& \dot{q}_{\mathrm{sg}, i}=\dot{q}_{\mathrm{sg}, i}^{\mathrm{gas}} \dot{q}_{\mathrm{sg}, i}^{\mathrm{solid}} \\
& \dot{q}_{\mathrm{ox}, i}=\dot{q}_{\mathrm{ox}, i}^{\mathrm{gas}} \dot{q}_{\mathrm{ox}, i}^{\mathrm{soli}} \\
& \dot{q}_{\mathrm{coag}, i}=\dot{q}_{\mathrm{coag}, i}^{\mathrm{fm}, \mathrm{gas}} \dot{q}_{\mathrm{coag}, i}^{\mathrm{fm}, \mathrm{solid}}+\dot{q}_{\mathrm{coag}, i}^{\mathrm{cl}, \mathrm{gas}} \dot{q}_{\mathrm{coag}, i}^{\mathrm{c} 1, \mathrm{solid}}+\dot{q}_{\mathrm{coag}, i}^{\mathrm{c} 2, \mathrm{gas}} \dot{q}_{\mathrm{coag}, i}^{\mathrm{c}, \mathrm{solid}}
\end{aligned}
$$


where the superscripts gas and solid correspond to the gaseous and soot dependence parts of each soot source term, which are detailed in AppendixA. It should be noted that the gaseous contribution parts depend only on $T, \rho$, the dynamic viscosity $\mu$, the pressure $P$, and the HACA-RC mechanism involved species concentrations [38, 39].

\subsection{Particle size distribution discretization}

Inside each section $i$, the soot volume fraction density $q(v)$ is considered constant and equal to $q_{i}=q\left(v_{i}^{\text {mean }}\right)$ with $v_{i}^{\text {mean }}=\left(v_{i}^{\min }+v_{i}^{\max }\right) / 2$. The volume particle number density $n(v)$ for each section is then evaluated for $v \in\left[v_{i}^{\min }, v_{i}^{\max }\right]$ through $n(v)=q_{i} / v$. The total soot volume fraction $f_{V}$ and particle number density $N_{\text {part }}$ are evaluated as:

$$
f_{V}=\int_{0}^{\infty} q(v) d v \quad \text { and } \quad N_{\text {part }}=\int_{0}^{\infty} n(v) d v .
$$

The particle size distribution discretization is done as follows:

- The first section is defined so that it contains all the nascent particles generated from the collisions of dimers of different sizes, depending on the number of PAHs considered,

- For $i \in \llbracket 2, N_{\text {sect }}-1 \rrbracket$, the volume intervals of the sections follow a geometrical progression:

$$
\begin{aligned}
& v_{i}^{\max }=v_{1}^{\max }\left(\frac{v^{\mathrm{MAX}}}{v_{1}^{\max }}\right)^{\frac{i-1}{N_{\mathrm{sect}-2}}} \\
& v_{i}^{\min }=v_{i-1}^{\max }
\end{aligned}
$$

- The last section can be considered as a "trash" section which contains very big unexpected soot particles from $v^{\mathrm{MAX}}$ to $v^{\mathrm{BIG}}$ and guarantees soot mass conservation. The value of $v^{\mathrm{BIG}}$ is chosen as an unattainable soot particle volume. The value of $v^{\operatorname{MAX}}$ corresponds to a characteristic volume of the expected biggest soot particles and is chosen as the maximum soot particle volume resolved accurately. 
This constructed discretization ensures that the volume interval $\left[v_{i}^{\min }, v_{i}^{\max }\right]$ described by each section $i$ is bigger than the sum of two volume intervals among the anterior sections.

\subsection{Morphological description}

Compared to the classical sectional models, in the present approach a soot particle is not always considered as spherical but its morphology depends on its size. For this, a soot particle of a given volume $v$ and surface $s$ is here considered as an aggregate composed of $n_{p}=s^{3} /\left(36 \pi v^{2}\right)$ primary spherical particles with a diameter $d_{p}=6 v / s$. The evolution of the particle surface $s$ is provided as a function of its volume $v$ as proposed in [33]:

$$
\left(s / s_{C_{2}}\right)=\left\{\begin{array}{c}
\left(v / v_{C_{2}}\right)^{2 / 3} \text { for } v<v_{1}, \\
\left(v / v_{C_{2}}\right)^{\theta(v) / 3} \text { for } v>v_{1}
\end{array}\right.
$$

with:

$$
\theta(v)=3.0 \cdot \frac{\cdot\left(\log \left(v / v_{1}\right)\right)+2 / 3 \cdot\left(\log \left(v_{1} / v_{C_{2}}\right)\right)}{\log \left(v / v_{C_{2}}\right)}
$$

where $v_{1}=320 \mathrm{~nm}^{3}$ denotes the volume beyond which a soot particle is no longer considered as spherical. $s_{C_{2}}$ and $v_{C_{2}}$ are respectively the surface and volume of a spherical molecule composed of two carbon atoms. Quantities $\theta(v), d_{p}(v)$ and $n_{p}(v)$ are supposed constant for each section $i$ and their values $\theta_{i}, d_{p, i}$ and $n_{p, i}$ are evaluated at $v_{i}^{\text {mean }}=\left(v_{i}^{\min }+v_{i}^{\max }\right) / 2$. This relation has been derived in [33] by fitting numerical results available in literature $[23,17]$ and is retained here since it is expected to impact not only surface reactions descriptions but also collisional phenomena via the value of the collisional diameter, function of the primary particles diameter $d_{p}$ and the number of primary particles $n_{p}$.

It should be noticed that for $v<v_{1}$, particles are spherical, so that $n_{p}=1$ and $d_{p}=(6 v / \pi)^{1 / 3}$.

\subsection{Treatment of dimers}

Dimer, an intermediate state for the polycyclic aromatic hydrocarbons (PAH) between the gaseous and the solid phases, is here obtained from the collision 
of two PAHs $[16,36]$. In the presented model, the dimerization of seven PAHs $\left(N_{\mathrm{PAH}}=7\right)$ with four or more aromatic rings are considered, from pyrene $\left(A_{4}\right)$ up to coronene $\left(A_{7}\right)$.

The dimerization production rate $\dot{Q}_{\mathrm{PAH}_{i}}^{\mathrm{d}}$ for a $\mathrm{PAH}_{i}$ is given by:

$$
\dot{Q}_{\mathrm{PAH}_{i}}^{\mathrm{d}}=2 v_{\mathrm{PAH}_{i}} \gamma_{i}\left(\frac{4 \pi k_{b} T}{m_{i}}\right)^{1 / 2} d_{\mathrm{PAH}_{i}}^{2}\left[\mathrm{PAH}_{i}\right]^{2} N_{A}^{2}
$$

where $d_{\mathrm{PAH}_{i}}$ is the diameter of a $\mathrm{PAH}_{i}$ particle (supposed spherical), $N_{A}$ the Avogadro number, $k_{b}$ the Boltzmann constant, $T$ the gas temperature, and $m_{i}$ the mass of the particle $\mathrm{PAH}_{i} . v_{\mathrm{PAH}_{i}}$ is the volume of a $\mathrm{PAH}_{i}$ evaluated as $v_{\mathrm{PAH}_{i}}=n_{\mathrm{PAH}_{i}} v_{C_{2}} / 2$ where $n_{\mathrm{PAH}_{i}}$ is the number of $C$ atoms of the $\mathrm{PAH}_{i}$ and $\gamma_{i}=C_{N} m_{i}^{4}$ is the sticking coefficient factor for $\mathrm{PAH}_{i}$ with $C_{N}$ a constant equal to $1.5 \cdot 10^{-11} g^{-4}[16]$.

To account for the multiple PAHs involved in dimerization process, an equivalent lumped $\mathrm{PAH}$ with mass fraction of $Y_{\mathrm{PAH}}$ is considered. Its mass fraction and total dimerization source term are evaluated as:

$$
\begin{aligned}
Y_{\mathrm{PAH}} & =\sum_{i=1}^{N_{\mathrm{PAH}}} Y_{\mathrm{PAH}_{i}} \\
\dot{Q}_{\mathrm{DIM}} & =\sum_{i=1}^{N_{\mathrm{PAH}}} \dot{Q}_{\mathrm{PAH}_{i}}^{\mathrm{d}}
\end{aligned}
$$

This lumped equivalent $\mathrm{PAH}$ leads to an equivalent dimer with a volume $v_{\mathrm{d}}$ evaluated as:

$$
v_{d}=2 \cdot \frac{\sum_{i=1}^{N_{\mathrm{PAH}}} \dot{Q}_{\mathrm{PAH}_{i}}^{\mathrm{d}}}{\sum_{i=1}^{N_{\mathrm{PAH}}} \dot{Q}_{\mathrm{PAH}_{i}}^{\mathrm{d}} /\left(2 v_{\mathrm{PAH}_{i}}\right)} .
$$

It will vary in the calculation depending on the local PAHs concentration.

For the calculation of the dimers number density $N_{d}$, a quasi-steady-state assumption is considered between their production from the gaseous phase and their consumption by nucleation and condensation [16, 36]:

$$
\dot{Q}_{\mathrm{DIM}}=\rho \sum_{i=1}^{N_{\mathrm{sect}}}\left(\dot{q}_{\mathrm{nu}, i}+\dot{q}_{\mathrm{cond}, i}\right) .
$$




\section{LES formalism for sooting non-premixed turbulent flames}

LES of sooting non-premixed turbulent flames requires the resolution of transport equations for both gaseous and solid phases. In this section, the novel features, mainly concerning the solid phase treatment will be provided, since the description of the reactive gaseous phase relies on classical LES formalism [43].

\subsection{Filtered soot sectional equations}

In the LES formalism, the filtered equation for soot section mass fraction is obtained applying a spatial filter to Eq. (1):

$$
\begin{aligned}
& \frac{\partial \bar{\rho} \widetilde{Y_{s, i}}}{\partial t}+\nabla \cdot\left(\bar{\rho} \widetilde{u_{i}} \widetilde{Y_{s, i}}\right)+\nabla \cdot\left(\overline{-\rho C_{\mathrm{th}} \frac{\nu}{T} \nabla T Y_{s, i}}\right) \\
& =\nabla \cdot\left(\bar{\rho} \widetilde{\mathbf{u} Y_{s, i}}-\bar{\rho} \widetilde{\mathbf{u} Y_{s, i}}\right)+\rho_{s} \dot{Q}_{s, i}
\end{aligned}
$$

where ${ }^{\sim}$ and $\sim$ denote the filtering and the density-weighted filtering operations, respectively.

Several terms in Eq. (12) are unclosed:

- $\mathbf{I}: J_{s, i}^{t}=\bar{\rho} \widetilde{\mathbf{u} Y_{s, i}}-\bar{\rho} \widetilde{\mathbf{u} Y Y_{s, i}}$ which represents the subgrid-scale soot section flux. This flux is modeled using a gradient assumption:

$$
J_{s, i}^{t}=-\bar{\rho} D_{s, i}^{t} \nabla \widetilde{Y_{s, i}}
$$

where $D_{s, i}^{t}=\nu^{\mathrm{sgs}} / \mathrm{Sc}_{s, i}^{\mathrm{sgs}}$ is the soot-section turbulent diffusivity, $\nu^{\mathrm{sgs}}$ is the turbulent viscosity obtained from the Wale model [44], and $\mathrm{Sc}_{s, i}^{\mathrm{sgs}}$ is the subgrid Schmidt number. In LES, this number is generally taken equal to the one obtained for a passive scalar, even in the case of small particles [? ]. However, it depends on the nature of the turbulent flow [? ]. In literature, it is generally considered constant, but it can be determined with a dynamic procedure [? ]. Here, it will be considered constant and equal to $\mathrm{Sc}_{s, i}^{\mathrm{sgs}}=0.6$, a value commonly retained in literature [? ]. 
- II : ${\overline{J_{s, i}}}^{\text {th }}=\overline{-\rho C_{\mathrm{th}} \frac{\nu}{T} \nabla T Y_{s, i}}$ which represents the filtered laminar thermophoresis flux closed as:

$$
{\overline{J_{s, i}}}^{\text {th }} \approx-\bar{\rho} C_{\mathrm{th}} \bar{\nu} \frac{\nabla \widetilde{T}}{\widetilde{T}} \widetilde{Y_{s, i}}
$$

This closure was originally proposed by Mueller and Pitsch [21, 29].

- III : $\overline{\dot{Q}_{s, i}}=\bar{\rho} \widetilde{\dot{q}_{s, i}}$ which will be closed through the subgrid model presented in Sec. 3.2. Following Eq. (2), it writes:

$$
\widetilde{\dot{q}_{s, i}}=\widetilde{\dot{q}_{\mathrm{nu}, i}}+\widetilde{\dot{q}_{\mathrm{cond}, i}}+\widetilde{\dot{q}_{\mathrm{sg}, i}}+\widetilde{\dot{q}_{\mathrm{ox}, i}}+\widetilde{\dot{q}_{\mathrm{coag}, i}}
$$

The filtered equation for soot mass fraction finally reads as:

$$
\begin{aligned}
& \frac{\partial \bar{\rho} \widetilde{Y_{s, i}}}{\partial t}+\nabla \cdot\left(\bar{\rho} \widetilde{\mathbf{u}} \widetilde{Y_{s, i}}\right)+\nabla \cdot\left(-\bar{\rho} C_{\mathrm{th}} \bar{\nu} \frac{\nabla \widetilde{T}}{\widetilde{T}} \widetilde{Y_{s, i}}\right) \\
& =\nabla \cdot\left(\bar{\rho} \frac{\nu^{\mathrm{sgs}}}{\mathrm{Sc}_{t}} \nabla \widetilde{Y}_{s, i}\right)+\rho_{s} \bar{\rho} \widetilde{\dot{q}_{s, i}}
\end{aligned}
$$

\subsection{Subgrid model for soot source terms}

In order to close the filtered soot source term, the subgrid model developed for the hybrid method of moments (HMOM) in [29] is here derived for the sectional method.

In this model, a filtered quantity $\widetilde{\psi}\left(\xi_{j}, \sigma_{i}\right)$, where $\xi_{j}$ and $\sigma_{i}$ are gaseous and soot scalars $\left(Y_{s, i}\right.$ for instance) respectively, is modeled with a joint subfilter $\operatorname{PDF} \widetilde{P}\left(\xi_{j}, \sigma_{i}\right)$ :

$$
\begin{aligned}
\widetilde{\psi}\left(\xi_{j}, \sigma_{i}\right) & =\iint \psi\left(\xi_{j}, \sigma_{i}\right) \widetilde{P}\left(\xi_{j}, \sigma_{i}\right) d \xi_{j} d \sigma_{i} \\
& =\iint \psi\left(\xi_{j}, \sigma_{i}\right) \widetilde{P}\left(\xi_{j}\right) P\left(\sigma_{i} \mid \xi_{j}\right) d \xi_{j} d \sigma_{i}
\end{aligned}
$$

As presented in Eq. (3) and detailed in AppendixA, the soot source terms can be written as a product of a first function depending only on the gaseous phase and a second function depending on the solid phase, so that the filtered soot source term is given by:

$$
\begin{array}{r}
\widetilde{\dot{q}_{\mathrm{p}, k}}\left(\xi_{j}, \sigma_{i}\right)=\iint \dot{q}_{\mathrm{p}, k}^{\mathrm{gas}}\left(\xi_{j}\right) \dot{q}_{\mathrm{p}, k}^{\mathrm{soot}}\left(\sigma_{i}\right) \\
\widetilde{P}\left(\xi_{j}\right) P\left(\sigma_{i} \mid \xi_{j}\right) d S_{g, j} d \xi_{j} d \sigma_{i}
\end{array}
$$


where the subscript $\mathrm{p}$ represents any subscript $\mathrm{nu}, \mathrm{cond}, \mathrm{sg}, \mathrm{ox}, \underset{\mathrm{coag}}{\mathrm{fm}}, \mathrm{coag}$ or coag.

Following Mueller and Pitsch [21], under the independency assumption between gaseous and soot quantities, this conditional soot distribution can be modeled by the corresponding marginal distribution:

$$
\widetilde{\dot{q}_{\mathrm{p}, k}}=\underbrace{\int \dot{q}_{\mathrm{p}, k}^{\text {gas }}\left(\xi_{j}\right) \widetilde{P}\left(\xi_{j}\right) d \xi_{j}}_{\left.\dot{q}_{\mathrm{p}, k}\right|_{\text {gas }}} \underbrace{\int \dot{q}_{\mathrm{p}, k}^{\mathrm{soot}}\left(\sigma_{i}\right) P\left(\sigma_{i}\right) d \sigma_{i}}_{\left.\dot{q}_{\mathrm{p}, k}\right|_{\text {soot }}}
$$

This assumption is valid if the time scales for the evolution of the thermochemical state are largely smaller than those of soot production source terms [21]. Then, the filtered soot source term reads:

$$
\widetilde{\dot{q}_{\mathrm{p}, k}}=\left.\left.\widetilde{\dot{q}_{\mathrm{p}, k}}\right|_{\text {gas }} \widetilde{\dot{q}_{\mathrm{p}, k}}\right|_{\text {soot }}
$$

where the themo-chemical and soot portions are now completely independent. The gaseous phase contribution $\left.\widetilde{\dot{q}_{\mathrm{p}, k}}\right|_{\text {gas }}$ can be modeled with any PDF approach classically developed for purely gaseous flames [42], which will be presented in Sec. 3.3 .

The solid phase contribution is modeled through a double-delta distribution function including a "non-sooting" mode and a "sooting" mode [29]:

$$
P\left(\sigma_{i}\right)=\omega \delta\left(\sigma_{i}\right)+(1-\omega) \delta\left(\sigma_{i}-\sigma_{i}^{*}\right)
$$

where $\omega$ is the subgrid soot intermittency and $\sigma_{i}^{*}$ the value of $\sigma_{i}$ for the sooting mode. The filtered value of $\sigma_{i}$ is evaluated respecting:

$$
\begin{aligned}
\widetilde{\sigma}_{i} & =\int \sigma_{i} P\left(\sigma_{j}\right) d \sigma_{j}=\sigma_{i}^{*}(1-\omega) \\
\Leftrightarrow & \sigma_{i}^{*}=\widetilde{\sigma}_{i} /(1-\omega)
\end{aligned}
$$

Then, the solid phase contribution can be expressed as:

$$
\begin{aligned}
\widetilde{\left.\dot{q}_{\mathrm{p}, k}\right|_{\text {soot }}}\left(\sigma_{i}\right) & =(1-\omega) \dot{q}_{\mathrm{p}, k}^{\mathrm{soot}}\left(\sigma_{i}^{*}\right) \\
& =(1-\omega) \dot{q}_{\mathrm{p}, k}^{\mathrm{soot}}\left(\frac{\widetilde{\sigma_{i}}}{1-\omega}\right)
\end{aligned}
$$


Equation (22) yields the following expression for the subgrid soot intermittency $\omega:$

$$
\omega=1-\frac{{\overline{\sigma_{i}}}^{2}}{\overline{\sigma_{i}^{2}}}
$$

As in [29], the particles number density $N_{\text {part }}$ is here chosen for the evaluation of $\omega$ :

$$
\omega=1-\frac{{\overline{N_{\text {part }}}}^{2}}{{\overline{N_{\text {part }}^{2}}}^{2} .}
$$

Then, in order to evaluate $\omega$, the filtered equations for $N_{\text {part }}$ and $N_{\text {part }}^{2}$ are added to the solid phase system of equations. The filtered equation for $\overline{N_{\text {part }}^{2}}$ is given as in [29]:

$$
\begin{aligned}
& \frac{\partial}{\partial t}\left(\overline{N_{\text {part }}^{2}}\right)+\nabla \cdot\left(\widetilde{\mathbf{u}} \overline{N_{\text {part }}^{2}}\right) \\
& =2 \bar{\rho}{m_{N} \dot{N}_{\text {part }}}^{2}-\overline{N_{\text {part }}^{2} \nabla \cdot \mathbf{u}}-\overline{N_{\text {part }}^{2} \nabla \cdot \mathbf{v}_{\mathbf{T}}} \\
& +\nabla \cdot\left(-\overline{\mathbf{v}_{\mathbf{T}} N_{\text {part }}^{2}}\right)+\nabla \cdot\left(\bar{\rho} \widetilde{\mathbf{u}} \frac{N_{\text {part }}^{2}}{\rho}-\overline{\rho \mathbf{u} \frac{N_{\text {part }}^{2}}{\rho}}\right)
\end{aligned}
$$

with $m_{N}=N_{\text {part }} / \rho$ and $\dot{N}_{\text {part }}$ the particles number density source term. This equation is closed here in analogy with Eq. (16) as follows:

- I : $J_{s, N_{\text {part }}^{2}}^{t}=\bar{\rho}\left(\widetilde{\left.\mathbf{u} \frac{N_{\text {part }}^{2}}{\rho}\right)}-\bar{\rho} \widetilde{\mathbf{u}}\left(\widetilde{\frac{N_{\text {part }}^{2}}{\rho}}\right)\right.$, with:

$$
\begin{aligned}
{\overline{J_{s, N_{\text {part }}^{2}}}}^{t} & =-\bar{\rho} D_{s, N_{\text {part }}^{2}}^{t} \nabla\left(\widetilde{\left.\frac{N_{\text {part }}^{2}}{\rho}\right)}\right. \\
& =-\bar{\rho} D_{s, N_{\text {part }}^{2}}^{t} \nabla\left(\frac{\widetilde{N_{\text {part }}^{2}}}{\bar{\rho}}\right)
\end{aligned}
$$

with $D_{s, N_{\text {part }}^{2}}^{t}=\nu^{\mathrm{sgs}} / \mathrm{Sc}_{N_{\text {part }}^{2}}^{\mathrm{sgs}}$. As for soot sections mass fractions, the turbulent Schmidt number $\mathrm{Sc}_{N_{\text {part }}^{2}}^{\mathrm{sgs}}$ is considered constant and equal to the common value of $0.6[?]$.

- II : ${\overline{J_{s, N_{\text {part }}^{2}}}}^{\text {th }}=\overline{\mathbf{v}_{\mathbf{T}} N_{\text {part }}^{2}}$ with:

$$
{\overline{J_{s, N_{\mathrm{part}}^{2}}}}^{\text {th }} \approx-\bar{\rho} C_{\mathrm{th}} \bar{\nu} \frac{\nabla \widetilde{T}}{\widetilde{T}}\left(\widetilde{N_{\mathrm{part}}^{2}}\right)=-\bar{\rho} C_{\mathrm{th}} \bar{\nu} \frac{\nabla \widetilde{T}}{\widetilde{T}} \frac{\overline{N_{\mathrm{part}}^{2}}}{\bar{\rho}}
$$


- III : $\overline{N_{\text {part }}^{2} \nabla \cdot \mathbf{u}}$ closed as in [29]:

$$
\overline{N_{\text {part }}^{2} \nabla \cdot \mathbf{u}}=\overline{N_{\text {part }}^{2}} \nabla \cdot \widetilde{\mathbf{u}}
$$

- IV : $\overline{N_{\text {part }}^{2} \nabla \cdot \mathbf{v}_{\mathbf{T}}} \approx \overline{N_{\text {part }}^{2}} \nabla \cdot \widetilde{\mathbf{V}_{\mathbf{T}}}$ in the same as for the term III. $\nabla \cdot \widetilde{\mathbf{v}_{\mathbf{T}}}$ is modeled by [29]:

$$
\nabla \cdot \widetilde{\mathbf{v}_{\mathbf{T}}} \approx \nabla \cdot\left(-C_{\mathrm{th}} \bar{\nu} \frac{\nabla \widetilde{T}}{\widetilde{T}}\right)
$$

- V : $\widetilde{m_{N} \dot{N}_{\text {part }}}$ which is closed with Eq. (17).

The final equation is given by:

$$
\begin{aligned}
& \frac{\partial \overline{N_{\text {part }}^{2}}}{\partial t}+\nabla \cdot\left(\widetilde{\mathbf{u}} \overline{N_{\text {part }}^{2}}\right)=2 \bar{\rho}{\dot{N_{\text {part }} m_{N}}}_{-\overline{N_{\text {part }}^{2}}} \nabla \cdot \widetilde{\mathbf{u}} \\
& -\overline{N_{\text {part }}^{2}} \nabla \cdot\left(-C_{\mathrm{th}} \bar{\nu} \frac{\nabla \widetilde{T}}{\widetilde{T}}\right)+\nabla \cdot\left(C_{\mathrm{th}} \bar{\nu} \frac{\nabla \widetilde{T}}{\widetilde{T}} \overline{N_{\text {part }}^{2}}\right) \\
& +\nabla \cdot\left(\bar{\rho} \frac{\nu^{\mathrm{sgs}}}{\mathrm{Sc}_{t}} \nabla\left(\widetilde{\frac{N_{\mathrm{part}}^{2}}{\rho}}\right)\right)
\end{aligned}
$$

The filtered equation for $N_{\text {part }}$ can be derived similarly:

$$
\begin{aligned}
& \frac{\partial \overline{N_{\text {part }}}}{\partial t}+\nabla \cdot\left(\widetilde{\mathbf{u}} \overline{N_{\text {part }}}\right)=\overline{\dot{N}_{\text {part }}}+\nabla \cdot\left(C_{\mathrm{th}} \bar{\nu} \frac{\nabla \widetilde{T}}{\widetilde{T}} \overline{N_{\text {part }}}\right) \\
& +\nabla \cdot\left(\bar{\rho} \frac{\nu^{\text {sgs }}}{\operatorname{Sc}_{t}} \nabla\left(\widetilde{\frac{N_{\text {part }}}{\rho}}\right)\right)
\end{aligned}
$$

The source term $\bar{N}_{\text {part }}$ is decomposed in five source terms, one for each phenomena involved in soot production:

$$
\overline{\dot{N}_{\text {part }}}=\overline{\dot{N}_{\text {nu }}}+\overline{\dot{N}_{\text {cond }}}+\overline{\dot{N}_{\text {sg }}}+\overline{\dot{N}_{\text {ox }}}+\overline{\dot{N}_{\text {coag }}}
$$

where for each phenomena $\mathrm{p}$, as the soot volume fraction density $q(v)$ is assumed constant inside each section, the corresponding source term is evaluated as:

$$
\begin{aligned}
\overline{\dot{N}_{\mathrm{p}}} & =\sum_{i=1}^{N_{\text {sect }}} \int_{v_{i}^{\min }}^{v_{i}^{\max }} \frac{\overline{\dot{Q}_{\mathrm{p}, i}}}{v_{i}^{\max }-v_{i}^{\min }} \frac{1}{v} d v \\
& =\sum_{i=1}^{N_{\text {sect }}} \frac{\overline{\dot{Q}_{\mathrm{p}, i}}}{v_{i}^{\max }-v_{i}^{\min }} \ln \left(\frac{v_{i}^{\max }}{v_{i}^{\min }}\right)
\end{aligned}
$$




\subsection{Subgrid model for gaseous quantities}

In this section, the subgrid models for the filtered gaseous quantities are detailed. They concern both the purely gaseous quantities as well as the gaseous contribution to the filtered soot source terms highlighted in Eq. (19).

Concerning the different fluxes, they are modeled with classical gradient assumptions, based on the Wale model [44] for the turbulent viscosity, and fixed values of 0.6 for both the subgrid Schmidt $\mathrm{Sc}^{\mathrm{sgs}}$ and Prandtl $\mathrm{Pr}^{\mathrm{sgs}}$ numbers.

Every gaseous thermochemical state is here described with the Flamelet/ Progress Variable (FPV) model [45], whose details are summarized for completeness. When neglecting heat losses, any gaseous thermochemical variable $\xi_{j}$ is obtained from solutions of steady non-premixed flamelet equations solved numerically as a function of the flame-normal spatial coordinate. The flamelets are computed for different strain rates allowing to describe the S-shape curve with stable and unstable branches. Following the FPV approach, the different variables are then parametrized as a function of the mixture fraction $Z$ and a normalized progress variable $C$ :

$$
\xi_{j}^{\mathrm{tab}}=\mathcal{F}(Z, C)
$$

where $\mathcal{F}$ represents the relationship obtained from the solution of the steady flamelet equations. The mixture fraction $Z$ is solved for each flamelet and corresponds to the solution of a passive scalar, with imposed boundary conditions equal to respectively $Z=0$ in the oxidizer side and $Z=1$ in the fuel side.

Radiative heat losses are accounted for by following the procedure of Ihme and Pitsch [46], who have extended the classical FPV model to account for heat losses due to radiation (radiation FPV model). The flamelet database is then augmented with solutions of unsteady flamelets computed by imposing the radiative source term to steady initial flamelets. To parametrize these unsteady 
flamelets, a heat loss parameter $H$ is added to the parametrization:

$$
\xi_{j}^{\mathrm{tab}}=\mathcal{G}(Z, C, H)
$$

where $\mathcal{G}$ represents the relationship obtained now with this new database.

The previous relationship is recast in terms of two quantities that uniquely identify each flamelet solution of the database: $\Lambda=C\left(Z_{0}\right)$ and $\Phi=H\left(Z_{0}\right)$, where $Z_{0}=0.064$. Each gaseous thermochemical quantity can then be retrieved as:

$$
\xi_{j}^{\mathrm{tab}}=\mathcal{G}(Z, C, H)=\mathcal{G}^{\star}(Z, \Lambda, \Phi) .
$$

Each gaseous filtered scalar quantity $\widetilde{\xi}_{j}^{\text {tab }}$ is expressed by:

$$
\widetilde{\xi}_{j}^{\mathrm{tab}}=\int \xi_{j}^{\mathrm{tab}}(Z, \Lambda, \Phi) \widetilde{P}(Z, \Lambda, \Phi) d Z d \Lambda d \Phi
$$

with $\widetilde{P}$, the density-weighted joint PDF. Equation (38) is then used to determine the gaseous part $\left.\widetilde{\dot{q}_{s, k}}\right|_{\text {gas }}$ of Eq. (19).

As in [46], we assume that the progress parameter $\Lambda$, the heat loss parameter $\Phi$ and the mixture fraction $Z$ are statistically independent. The joint PDF can then be expressed in terms of the marginal distributions of each parameter:

$$
\widetilde{P}(Z, \Lambda, \Phi)=\widetilde{P}_{Z}(Z) P_{\Lambda}(\Lambda) P_{\Phi}(\Phi)
$$

with $P$, the non-weighted joint PDF.

A $\beta$-PDF is used to model the mixture fraction distribution, implemented following the second-order numerical approach of Lien et al. [47]. The statistical distributions of the reaction progress and heat loss parameters are represented by a Dirac function. Then, the joint subfilter gaseous PDF can be expressed by:

$$
\widetilde{P}(Z, \Lambda, \Phi)=\beta\left(Z ; \widetilde{Z}, S_{Z}\right) \delta(\Lambda-\widetilde{\Lambda}) \delta(\Phi-\widetilde{\Phi}) .
$$

where $S_{Z}$ is the mixture fraction segregation factor.

Each gaseous thermochemical variable, except for the PAH concentration which requires a special treatment that will be presented in Sec. 3.4, can then 
be retrieved in a precomputed 4-D table, and can be expressed as:

$$
\widetilde{\xi}_{j}^{\mathrm{tab}}=\widetilde{\xi}_{j}^{\mathrm{tab}}\left(\widetilde{Z}, S_{Z}, \widetilde{\Lambda}, \widetilde{\Phi}\right) \equiv \widetilde{\xi}_{j}^{\mathrm{tab}}\left(\widetilde{Z}, S_{Z}, \bar{C}, \bar{H}\right) .
$$

To retrieve the variables of the table, the passive scalar $\widetilde{Z}$ is directly transported together with the other radiation FPV-state-variables: the variance of the mixture fraction $\widetilde{Z^{\prime \prime 2}}$, the enthalpy $\widetilde{h}$ and the progress variable $\widetilde{Y_{C}}$. Then, $S_{Z}, \bar{C}$ and $\bar{H}$ are then evaluated as:

$$
\begin{aligned}
S_{Z} & =\frac{\widetilde{Z^{\prime \prime 2}}}{\widetilde{Z}(1-\widetilde{Z})} \\
\bar{H} & =\frac{\widetilde{h}-\widetilde{h^{\mathrm{rad}}}\left(Z, S_{Z}\right)}{\widetilde{h^{\mathrm{adiab}}}\left(Z, S_{Z}\right)-\widetilde{h^{\mathrm{rad}}}\left(Z, S_{Z}\right)} \\
\bar{C} & =\frac{\widetilde{Y_{C}}-\widetilde{Y_{C}^{\mathrm{f}}}\left(Z, S_{Z}\right)}{\widetilde{Y_{C}^{\mathrm{eq}}}\left(Z, S_{Z}\right)-\widetilde{Y_{C}^{\mathrm{f}}}\left(Z, S_{Z}\right)}
\end{aligned}
$$

where $h^{\text {adiab }}$ is the enthalpy of the adiabatic flamelet, $h^{\text {rad }}$ is the enthalpy of the flamelet presenting the maximum of radiation heat losses. $Y_{C}$ is the nonnormalized progress variable defined as a weighted sum of species mass fractions with $Y_{C}^{\mathrm{eq}}$ its value for the lowest strain rate flamelet on the stable branch of the $\mathrm{S}$ curve and $Y_{C}^{\mathrm{f}}$ its frozen value when chemical reactions are neglected.

\subsection{PAH model}

Because of their long chemical time scales, PAHs may not lie on the flamelet manifold and cannot be treated as the other gaseous characteristics. Several approaches have been proposed in literature, from the transport of one unique lumped PAH representing all the classes of PAHs [21], to the transport of several lumped PAHs, each one corresponding to each class of PAHs [19]. Here, as a first step, the approach of Mueller and Pitsch [21] with one unique lumped PAH is used for modeling the unsteady effects of strain rate and transport on PAH production. The spatially-filtered transport equation for the lumped PAH mass fraction $\widetilde{Y_{\mathrm{PAH}}}$ is given by:

$$
\begin{aligned}
& \frac{\partial \bar{\rho} \widetilde{Y}_{\mathrm{PAH}}}{\partial t}+\nabla \cdot\left(\bar{\rho} \widetilde{\mathbf{u}} \widetilde{Y}_{\mathrm{PAH}}\right)=\nabla \cdot\left(\bar{\rho} \widetilde{\mathbf{u}} \widetilde{Y}_{\mathrm{PAH}}\right. \\
& \left.-\bar{\rho} \widetilde{\mathbf{u} Y_{\mathrm{PAH}}}\right)+\overline{\nabla \cdot\left(\rho D_{\mathrm{PAH}} \nabla Y_{P A H}\right)}+\bar{\rho} \dot{Q}_{\mathrm{PAH}}
\end{aligned}
$$


Several terms are unclosed in Eq. (45) and require some modeling efforts:

- $\mathbf{I}: J_{\mathrm{PAH}}^{t}=\bar{\rho} \widetilde{\mathbf{u} Y_{\mathrm{PAH}}}-\bar{\rho} \widetilde{\mathbf{u}} \widetilde{\mathrm{PAH}}$ closed as:

$$
J_{\mathrm{PAH}}^{t}=-\bar{\rho} D_{\mathrm{PAH}}^{t} \nabla \widetilde{Y_{\mathrm{PAH}}}
$$

with $D_{\mathrm{PAH}}^{t}=\nu^{\mathrm{sgs}} / \mathrm{Sc}_{\mathrm{PAH}}^{\mathrm{sgs}}$. As for soot sections mass fractions, the turbulent Schmidt number $\mathrm{Sc}_{\mathrm{PAH}}^{\mathrm{sgs}}$ is considered constant and equal to its common value of $0.6[?]$,

\section{- II:}

For the lumped species, the source term $\widetilde{\dot{Q}_{\text {PAH }}}$ can be split into three different terms: the chemical production term $\left(\dot{Q}_{\mathrm{PAH},+}\right)$, the chemical consumption term $\left(\dot{Q}_{\mathrm{PAH},-}\right)$, which is linear with the species concentration, and the dimerization source term $\left(\dot{Q}_{\mathrm{DIM}}\right)$, which is quadratic with the species concentration. The corresponding decomposition can be written as:

$$
\begin{aligned}
\widetilde{\dot{Q}_{\mathrm{PAH}}} & =\widetilde{\dot{Q}_{\mathrm{PAH},+}}+\widetilde{\left(\frac{\dot{Q}_{\mathrm{PAH},-}}{Y_{\mathrm{PAH}}}\right) Y_{\mathrm{PAH}}} \\
& =\overbrace{\left(\frac{\dot{Q}_{\mathrm{DIM}}}{Y_{\mathrm{PAH}}^{2}}\right) Y_{\mathrm{PAH}}^{2}}
\end{aligned}
$$

To close the second and third terms of this equation, the following relationship between the transport equation model and the radiation FPV model is used [21]:

$$
\begin{aligned}
& \overline{\dot{Q}}_{\mathrm{PAH}}={\widetilde{\dot{Q}_{\mathrm{PAH},+}}}^{\mathrm{tab}}+{\widetilde{\dot{Q}_{\mathrm{PAH},-}}}^{\mathrm{tab}}\left(\frac{\widetilde{Y_{\mathrm{PAH}}}}{\widetilde{Y_{\mathrm{PAH}}}{ }^{\mathrm{tab}}}\right) \\
& +{\widetilde{\dot{Q}_{\mathrm{DIM}}}}^{\mathrm{tab}}\left(\frac{\widetilde{Y_{\mathrm{PAH}}}}{\widetilde{Y_{\mathrm{PAH}}}{ }^{\mathrm{tab}}}\right)^{2} .
\end{aligned}
$$

For the evaluation of the lumped source terms from the table $\widetilde{\hat{Q}_{\mathrm{PAH},+}}$ tab ${\widetilde{Q_{\mathrm{PAH},-}}}^{\mathrm{tab}},{\widetilde{\dot{Q}_{\mathrm{DIM}}}}_{\mathrm{tab}}^{\mathrm{tab}}$ and the value of ${\widetilde{Y_{\mathrm{PAH}}}}^{\mathrm{tab}}$, they are computed by sum- 
ming their respected values obtained for the $N_{\mathrm{PAH}}$ selected PAHs:

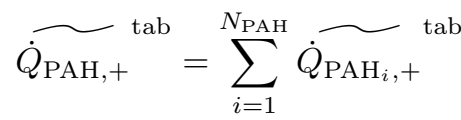

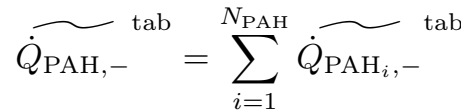

$$
\begin{aligned}
& {\widetilde{\dot{Q}_{\mathrm{DIM}}}}^{\mathrm{tab}}=\sum_{i=1}^{N_{\mathrm{PAH}}} \overbrace{\dot{Q}_{\mathrm{PAH}}^{\mathrm{d}}}^{\mathrm{tab}} \\
& {\widetilde{Y_{\mathrm{PAH}}}}^{\mathrm{tab}}=\sum_{i=1}^{N_{\mathrm{PAH}}}{\widetilde{Y_{\mathrm{PAH}_{i}}}}^{\mathrm{tab}}
\end{aligned}
$$

- III : $\overline{\nabla \cdot\left(\rho D_{\mathrm{PAH}} \nabla Y_{P A H}\right)}$ :

In the limit of very large Reynolds number, the appropriate diffusion model used in the flamelet computation is a unity Lewis number model because of high turbulent diffusivities compared with the molecular ones [48]. Unity Lewis numbers have then been assumed for all the PAHs accordingly for the construction of the table given the target simulation which is a turbulent jet flame.

Nonetheless, the study by Pitsch [49] on differential diffusion in turbulent diffusion flames highlighted that such effects are not always negligible and are even significant in the close vicinity of the jet nozzle where the flow is weakly turbulent such as in the studied configuration. In such regions, one should then take into account the high Lewis numbers for PAHs. The production of PAHs and soot particles has indeed been shown to be sensitive to the PAHs and soot diffusion model (non-unity or unity Lewis number) in direct numerical simulations of a temporal mixing layer [20] and in RANS studies of turbulent jet diffusion flames [? ? ? ]. Non-negligible effects of PAHs high Lewis numbers in weakly turbulent zones can therefore also be expected in the large-eddy simulation of the considered jet flame close to the jet exit.

Consequently, the choice of the diffusion model for PAHs can be strongly important and is not straightforward: unity Lewis model assumption ev- 
erywhere or non-unity Lewis model assumption can lead to large errors in LES of sooting flames. Such an issue is a challenging task in the modelling community that remains to be tackled. An alternative compromise is considered here to partially account for differential diffusion effects by retaining the real molecular diffusivities $D_{\mathrm{PAH}_{i}}$ in the transport equations for PAHs:

$$
\overline{\nabla \cdot\left(\rho D_{\mathrm{PAH}} \nabla Y_{P A H}\right)}=\nabla \cdot\left(\bar{\rho} \overline{D_{\mathrm{PAH}}} \nabla \widehat{Y_{P A H}}\right)
$$

The competition between molecular diffusion and turbulent transport is then captured at the resolved scales only. However, it should be noticed that an inconsistency appears since the flamelet table has been generated with unity Lewis numbers. This is tempered by the previously described relaxation model, which allows the PAHs deviation from the flamelet manifold.

Finally, since a lumped PAH is considered, the corresponding lumped diffusion coefficient $\overline{D_{\mathrm{PAH}}}$ is calculated as:

$$
\overline{D_{\mathrm{PAH}}}=\frac{\overline{D_{\mathrm{PAH}_{i}}} \nabla Y_{\mathrm{PAH}_{i}}}{\nabla Y_{\mathrm{PAH}}} .
$$

\subsection{Radiative heat transfer modeling}

Due to its high dependence on temperature, radiation plays an important role in jet flames [46]. Its role is even more significant in the case of sooting flames and the quality of the radiation model can have major effects on the prediction of soot and gaseous quantities $[50,51]$. As a first approach, an optically-thin radiation model is used in the present work. For the gaseous phase, Planck mean absorption coefficients are used [52] and $\mathrm{CO}_{2}, \mathrm{H}_{2} \mathrm{O}$ and $\mathrm{CO}$ are considered as the main contributing gaseous species to the radiative energy transfer. For the soot particles, the Rayleigh scattering assumption is considered. A Planck mean absorption coefficient $\kappa_{\text {soot }}^{\text {Planck }}(T)$ [53] is used with:

$$
\kappa_{\text {soot }}^{\text {Planck }}(T)=3.83 \frac{C_{0} f_{V} T}{C_{2}}
$$


with $C_{0}=\frac{36 \pi a b}{\left(a^{2}-b^{2}+2\right)^{2}+4 a^{2} b^{2}}$ and $C_{2}=h c / k_{B}$ the second Planck constant. The index of soot particles is taken equal to $m=a-i b=1.57-0.56 i$ [54].

Then, the total radiative source term $P_{\text {rad }}$ is expressed by:

$$
P_{\mathrm{rad}}=-4 \sigma\left(\sum_{i}\left(a_{\mathrm{Pl}, i} p_{i}\right)+\kappa_{\text {soot }}^{\mathrm{Planck}}\right) \widetilde{T}^{4}
$$

where $a_{\mathrm{Pl}, i}$ is the Planck mean absorption coefficient of species $i$ calculated as in [52] and $p_{i}$ is the partial pressure of species $i$.

\section{Application to the simulation of a turbulent flame}

The LES formalism described in Sec. 3 is applied here to the simulation of soot production in a 3-D turbulent ethylene/air flame.

\subsection{Experimental configuration}

The configuration chosen for the simulation is the turbulent non-premixed pure ethylene/air diffusion flame which has been extensively characterized experimentally at Sandia [55]. This configuration corresponds to a turbulent jet with Reynolds $R e_{D}=20000$, based on the fuel injector diameter of the main jet $D=3.2 \mathrm{~mm}$. The corresponding bulk velocity is $v_{\text {fuel }}=54.7 \mathrm{~m} / \mathrm{s}$.

The main jet tube presents an outer diameter of $4.6 \mathrm{~mm}$ and is surrounded by another tube with an inner diameter of $15.2 \mathrm{~mm}$, and an outer diameter of $19.1 \mathrm{~mm}$. Both tubes are surrounded by 64 pilot flames for the stabilization of the flame. These pilot flames are fed by ethylene/air mixture with an equivalence ratio of $\phi=0.9$. The global mass flow rate of the 64 pilot injectors is equal to $1.77 \times 10^{-4} \mathrm{~kg} / \mathrm{s}$ and their total heat release corresponds to only $2 \%$ of the heat release of the main jet. The pilot flames size, number and spacing have been chosen such that they produce a uniform flow rate of flame products across the burner exit plane. Finally, a coflow of air at $v_{\text {air }}=0.6 \mathrm{~m} / \mathrm{s}$ surrounds the whole pilot flames.

The inlet temperatures of all the flows, except the pilot, are equal to $294 \mathrm{~K}$. 
For the pilot flame, an adiabatic flame temperature equal to $2296 \mathrm{~K}$ for the corresponding equivalence ratio has been imposed.

Different sets of experimental data are used for the comparison with numerical results:

- CARS temperature and $X_{\mathrm{O}_{2}} / X_{N_{2}}$ measures from [62],

- PLIF OH and LII soot volume fractions measures from [64],

- Axial profile of soot intermittency measures from [63].

\subsection{Choice of the gaseous phase and table generation}

The detailed kinetic scheme KM2 [56] has been retained to solve the steady and unsteady equations for the 1-D counterflow flames for the flamelet database with the REGATH package [57]. The mechanism involves 202 species and 1351 reactions and has been validated for $C_{0}-C_{4}$ fuels and for the estimation of PAH up to coronene [56]. The dimerization source term defined in Eq. (8) is added to each $\mathrm{PAH}$ chemical source term involved in dimerization process in order to take into account in the flamelet database, the PAH removal due to nucleation and condensation processes. Unity Lewis diffusion model is used for the generation of the flamelet database. A validation of the sectional model coupled with this kinetic scheme has been done in 1-D laminar premixed and diffusion configurations in a previous work [33].

The progress variable $Y_{C}$ is chosen equal to

$$
\begin{array}{r}
Y_{C}=\left(\frac{Y_{\mathrm{CO}_{2}}}{W_{\mathrm{CO}_{2}}}+\frac{Y_{\mathrm{H}_{2} \mathrm{O}}}{W_{\mathrm{H}_{2} \mathrm{O}}}+\frac{Y_{C O}}{W_{C O}}-3 \frac{Y_{\mathrm{CH}_{4}}}{W_{\mathrm{CH}_{4}}}\right) / \\
\left(1 / W_{\mathrm{CO}_{2}}+1 / W_{\mathrm{H}_{2} \mathrm{O}}+1 / W_{\mathrm{CO}}-3 / W_{\mathrm{CH}_{4}}\right),
\end{array}
$$

such that it uniquely maps each flamelet for the studied case and the considered kinetic scheme. 


\subsection{Numerical setup}

The lookup table was discretized with $100 \times 20 \times 100 \times 20$ grid points in

the directions $\widetilde{Z} \times S_{Z} \times \bar{C} \times \bar{H}$, respectively. The model presented in Sec. 3 was implemented in the code AVBP [58]. This parallel CFD code, developed at CERFACS and IFPEN, solves the three-dimensional compressible NavierStokes equations on unstructured meshes. The third-order in space and time finite element TTGC scheme [59] is retained for this simulation. Navier-Stokes Characteristic Boundary Conditions (NSCBC) [60] are used to prescribe the boundary conditions. The Tabulated Thermochemistry for Compressible flows formalism (TTC) is used [61]. Flamelets, and hence the flamelet table, are computed with a low Mach-number assumption which results in neglecting compressible effects in the combustion model. This approximation is valid in the studied case. However, using the enthalpy given by the set of compressible transport equations directly in Eq. (43) would wrongly impress variations in the tabulated quantities (species mass fractions, $\cdots$ ) associated to the captured acoustic waves. Therefore, an additional equation for the enthalpy with a lowMach number approximation is here transported. The corresponding field $\widetilde{h}$ is fed to Eq. (43). For the solid phase, 25 sections are transported to describe the particle size distribution describing particles with volumes comprised between $v^{\mathrm{MIN}}=0.7 \mathrm{~nm}^{3}$ and $v^{\mathrm{MAX}}=5 \times 10^{9} \mathrm{~nm}^{3}$. This value is a compromise between accuracy and limited CPU over-cost, based on a convergence study on 1-D laminar flames showing that 25 sections are sufficient for the prediction of the soot volume fraction.

Fully-developed pipe flow mean and RMS radial profiles have been obtained from a preliminary computation in order to impose the inlet turbulent boundary conditions.

The computational domain (Fig. 1) is composed of three inlets, the walls and one outlet. It extends to $312.5 \mathrm{D}$ downstream, $8 \mathrm{D}$ upstream of the nozzle and $94 \mathrm{D}$ in the radial direction. The 64 pilot flames are modeled with a single concentric flow with the uniform mass flow rate. The mesh contains $10 \mathrm{M}$ cells $/ 1.7 \mathrm{M}$ nodes and the typical cell size at the jet exit is $\Delta x \approx 0.20 \mathrm{~mm}$. 


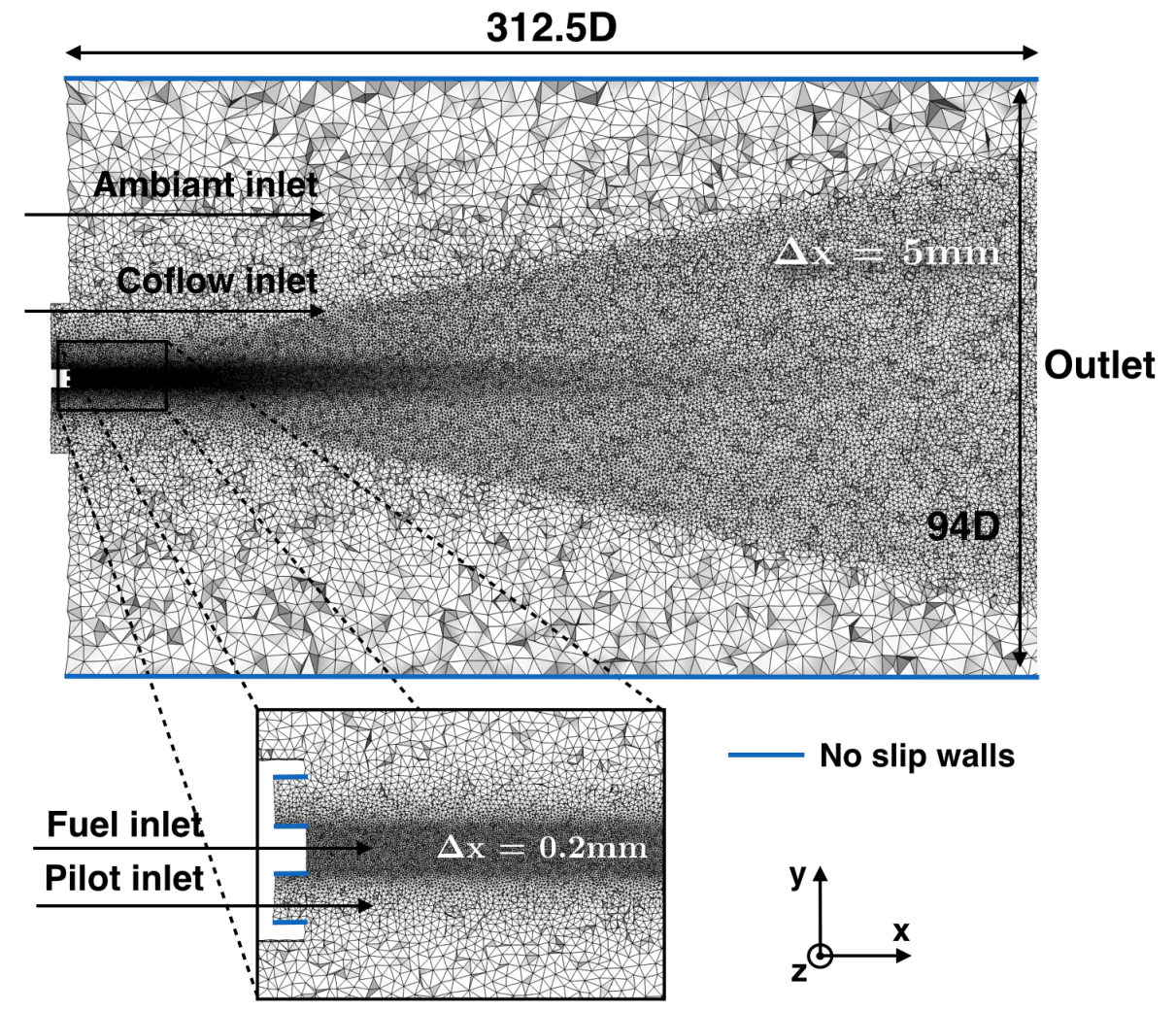

Figure 1. Longitudinal cut of the cylindrical computational domain.

This simulation has been performed using a Bull cluster equipped with Intel E5-2680 processors with a total computational time (including averaging time) of 750 thousands of CPU hours. The averages have been done over $250 \mathrm{~ms}$ of physical time.

\subsection{Numerical validation: comparison with experimental results}

In order to validate the LES approach, the numerical results are compared to the available experimental data. Concerning the gaseous phase, Fig. 2 (top) shows a comparison of radial mean and root mean square (RMS) temperature profiles with experiments at $x / D=134$ [62]. In Fig. 2 (bottom), results for mean and RMS of $X_{O_{2}} / X_{N_{2}}$ ratio are presented for the same height. Good 
prediction of the mixture and temperature is obtained. Small overestimation of temperature at the centerline is observed and peaks of radial temperature and $X_{O_{2}} / X_{N_{2}}$ ratio RMS are slightly underestimated. Several aspects can affect the quality of these results: the predicted turbulent mixing, the turbulent combustion model and also the radiation modeling which is important because of its coupling with temperature. Moreover, it should be reminded that this set of experimental data [62] comes from measures in altitude where the pressure is about $15 \%$ lower than the one used in our numerical setup (1atm). The overall agreement is satisfactory and good predictions of the gaseous phase are essential for the prediction of the source terms of the solid phase evolution.
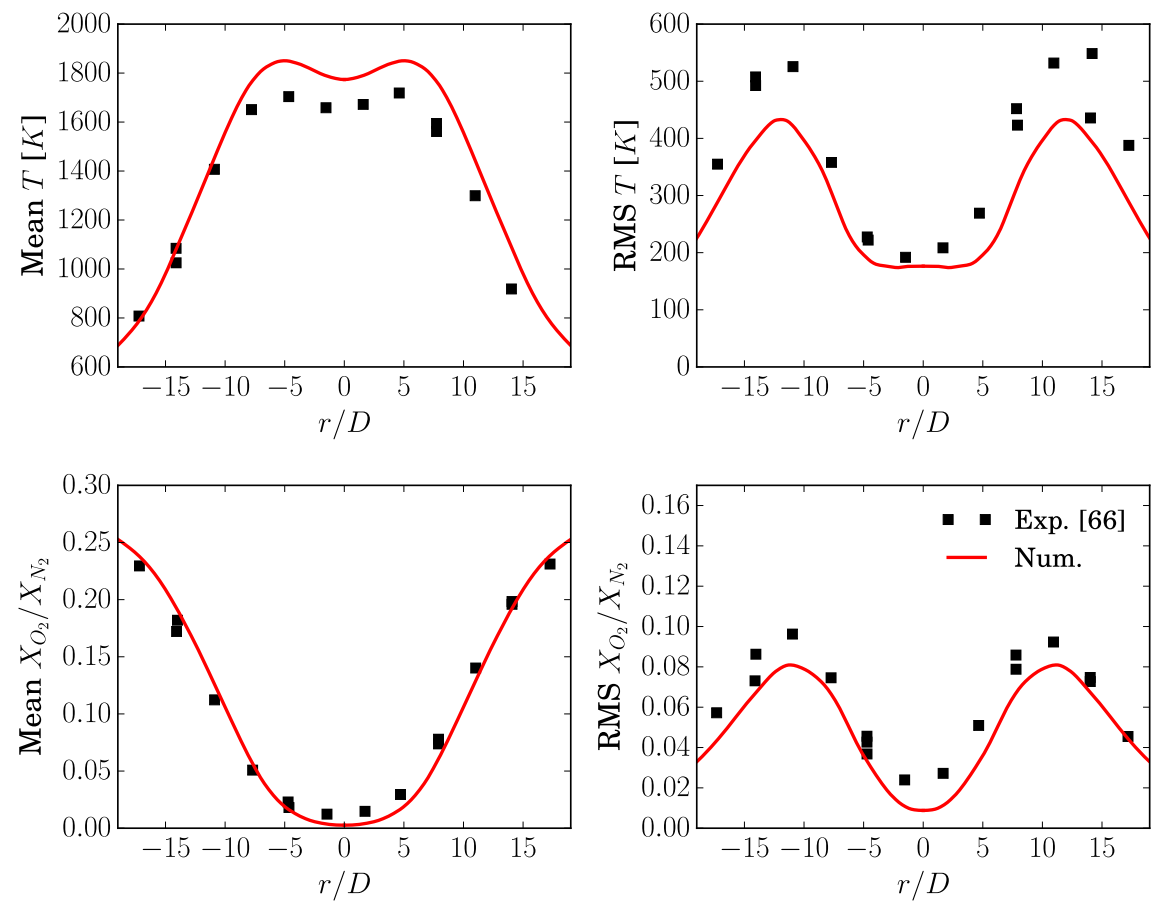

Figure 2. Comparison of mean and RMS temperature and $X_{\mathrm{O}_{2}} / X_{N_{2}}$ radial profiles between numerical (line) and experimental results (symbols) at $x / D=134$.

In addition, Fig. 3 shows a comparison of mean $\mathrm{OH}$ radial profiles at differ- 
ent heights above the burner between predictions and measurements [64]. The experimental data being non-quantitative, experimental and numerical results are here normalized by their respective maximum values for each height above the burner. The obtained agreement confirms a good prediction of the position of the flame front and of the mean flame brush, necessary to correctly locate soot oxidation phenomena.
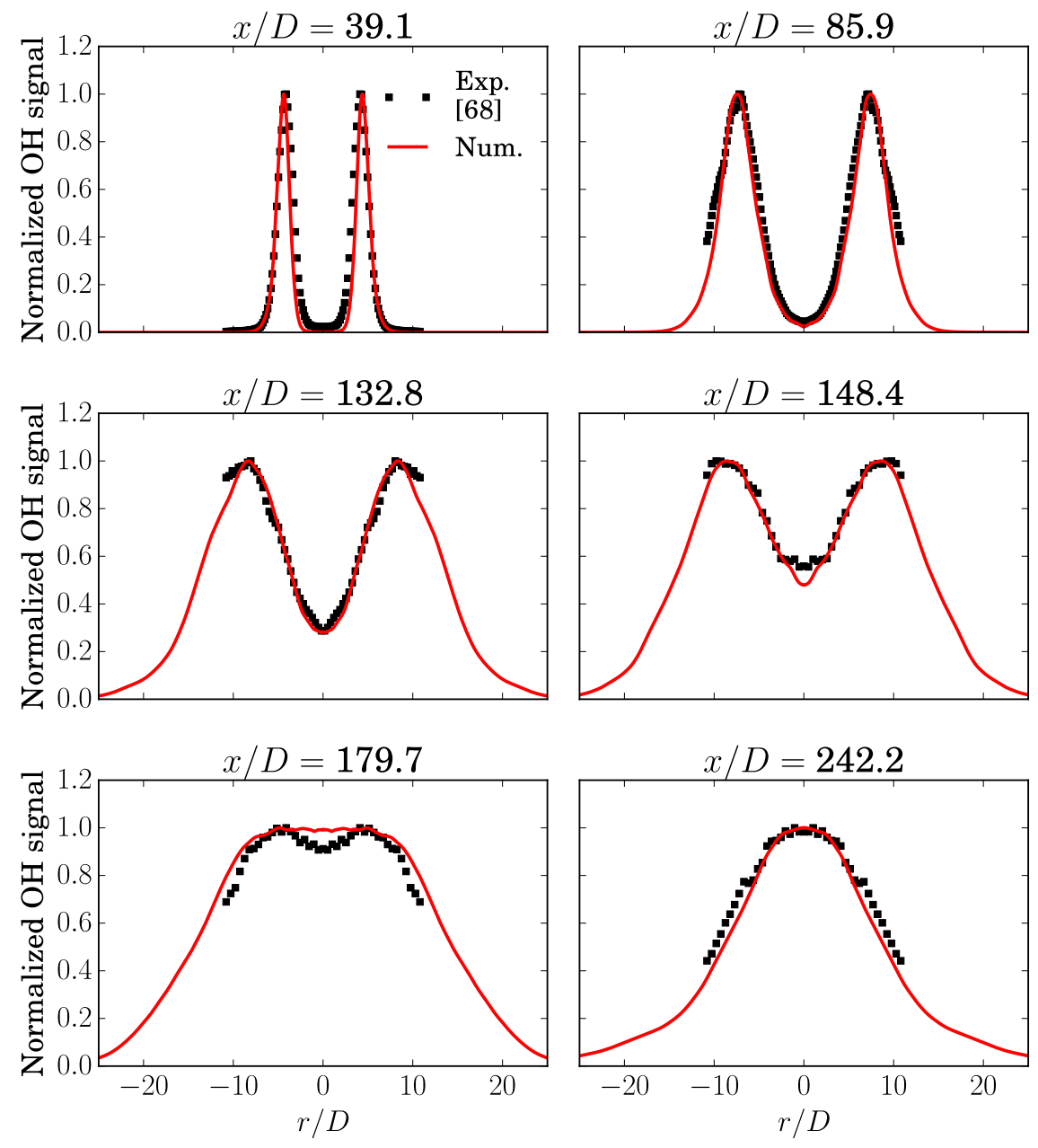

Figure 3. Normalized mean $\mathrm{OH}$ radial profiles at different heights above the burner: numerical results (line) are compared to experiments (symbols). 
In order to validate the proposed approach for sooting turbulent flames, it is firstly possible to quantify the resolved temporal soot intermittency. This quantity is defined experimentally at each point as the probability of finding an instantaneous value of $f_{V}$ lower than $0.03 \mathrm{ppm}^{1}$. Figure 4 shows a comparison of numerically-resolved soot intermittency and experimental probe-resolved soot intermittency along the flame centerline as a function of the axial position. It can be seen that the model reproduces well this quantity even if numerical results seem slightly translated upstream so that at $x / D>150$ soot presence is detected experimentally whereas no more soot particles are obtained in the simulation. Globally, these results seem to confirm a good prediction of soot particles production dynamics.

\footnotetext{
${ }^{1}$ It is important to notice that the temporal soot intermittency does not correspond to the subgrid intermittency previously defined in Eq. (25).
} 


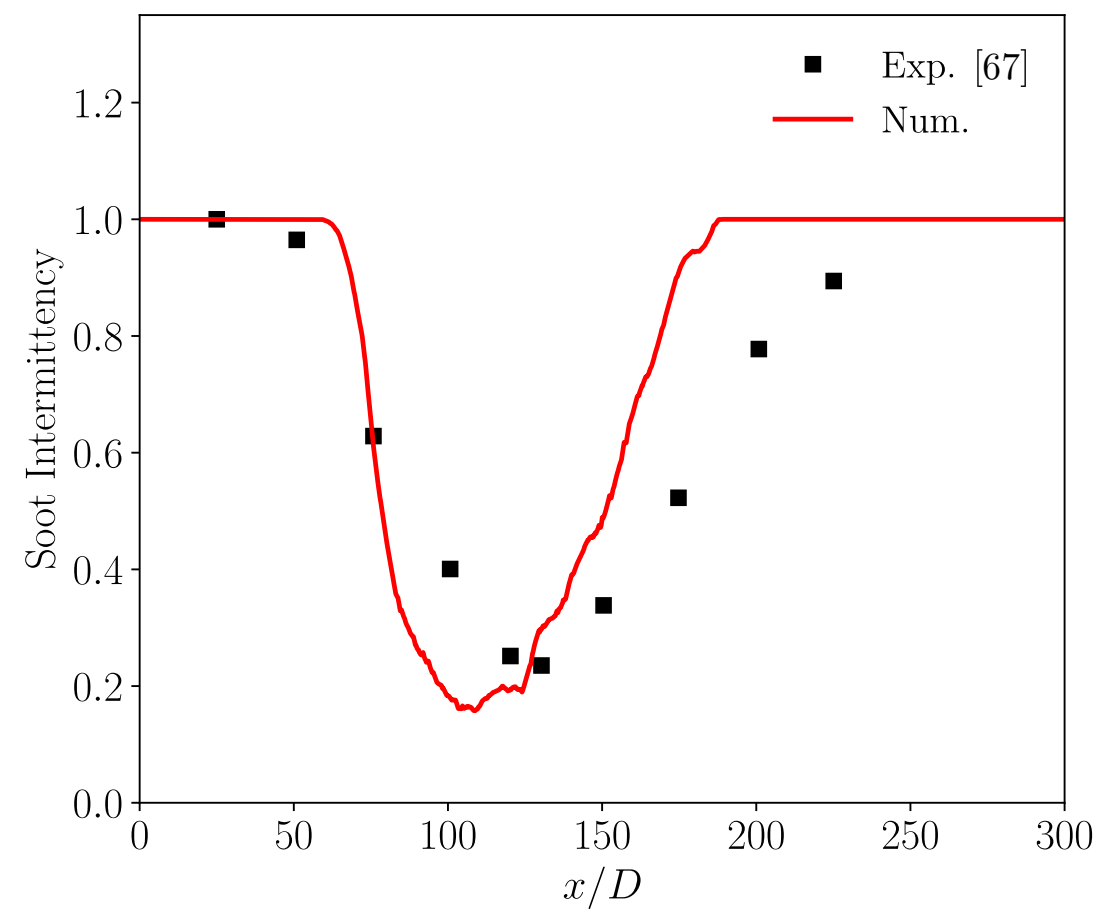

Figure 4. Comparison of numerical (line) and experimental (symbols) soot intermittency axial profiles.

Axial mean soot volume fraction profile is compared with experiments in Fig. 5 [64]. A reasonable agreement of soot production is obtained, but the peak soot volume fraction is overpredicted by a factor two. Soot destruction is also predicted too early compared to experiments. In literature, similar results on other sooting jet flames have already been observed by [21], whereas a previous work based on the DQMOM model presented an underestimation of $f_{V}$ for the currently studied flame [22]. As a consequence, it can be said that the present prediction of soot volume fraction is reasonable compared to the state-of-the-art in large eddy simulations of soot production. 


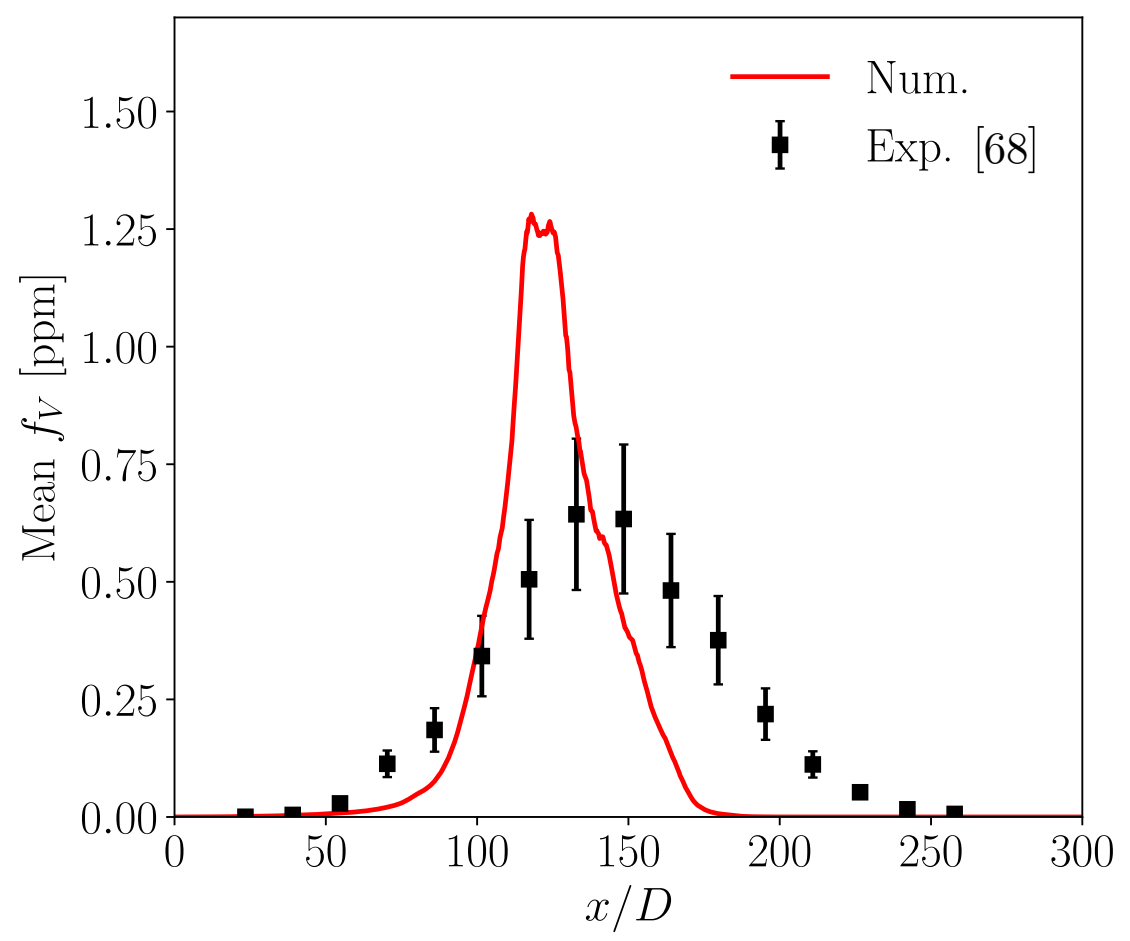

Figure 5. Soot volume fraction mean axial profiles: comparison between experimental (symbols) and numerical (line) data.

Figure 6 compares radial mean profiles for soot volume fractions at different heights above the burner. As for the axial profiles, an overprediction of soot volume fraction magnitude is obtained between $\mathrm{x} / \mathrm{D}=110$ and $\mathrm{x} / \mathrm{D}=140$. The width of the soot volume fraction zone is underpredicted.

Figure 7 compares relative radial RMS profiles of $f_{V}$ for the same heights. The relative radial RMS corresponds to the soot volume fraction RMS divided by the mean soot volume fraction. This comparison enables to focus only on the prediction of high RMS zone of soot volume fraction production. Predictions of the magnitude and position of high relative RMS of soot production are quite reasonable compared to experiments. Then, it can be concluded that despite 
the discrepancies on soot volume fraction magnitude prediction, the temporal dynamics of soot production are well predicted.
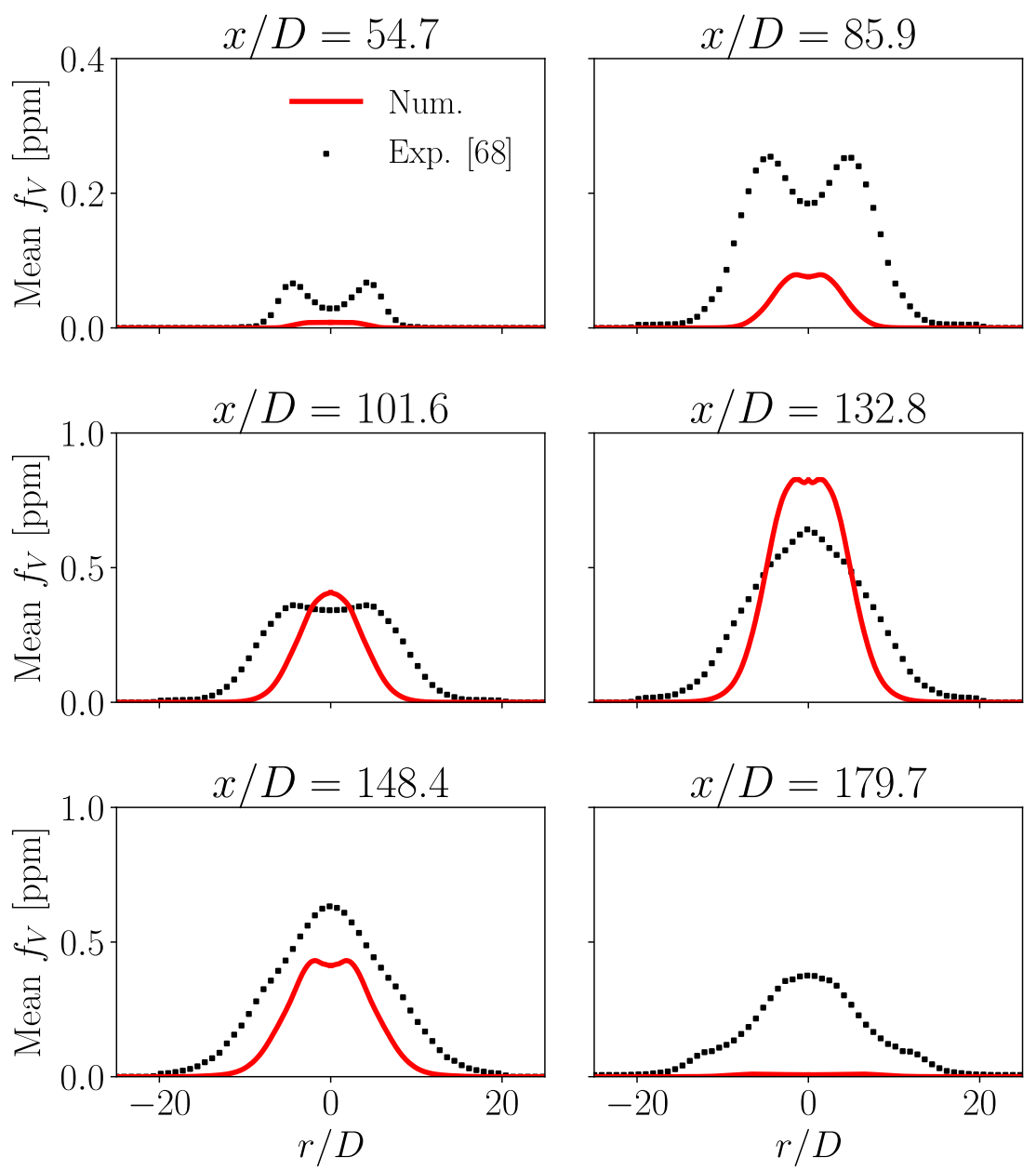

Figure 6. Soot volume fraction mean radial profiles at different heights above the burner: comparison between experimental (symbols) and numerical (line) data. 

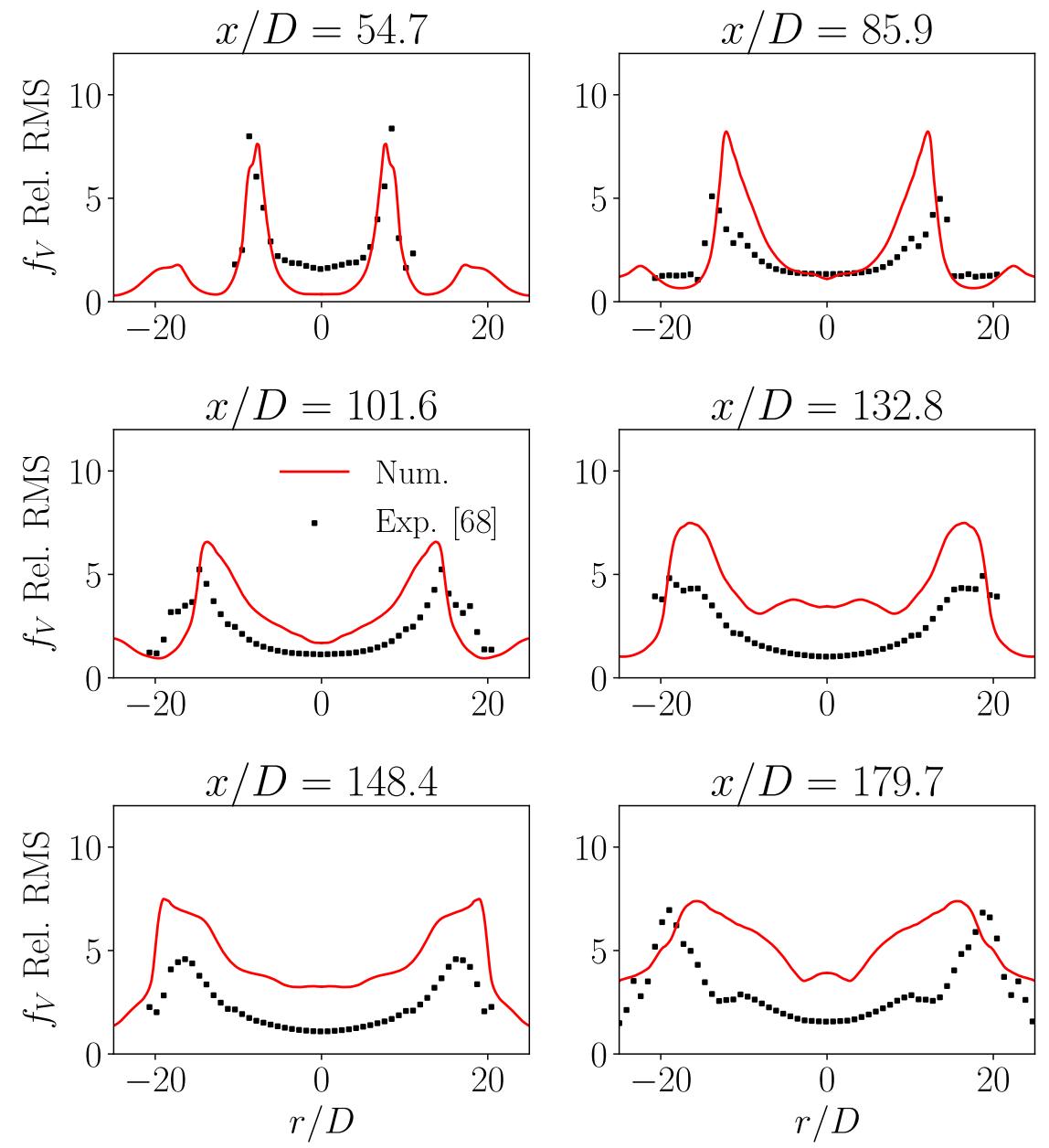

Figure 7. Relative soot volume fraction RMS radial profiles at different heights above the burner: comparison between experimental (symbols) and numerical (line) data.

In conclusion, despite the fact that errors in soot volume fraction magnitude and position predictions are still present with the proposed sectional method, this simulation belongs to the state-of-the-art in terms of soot production prediction. In addition, it provides the access to the particle size distribution evolution, enabling new analysis of soot particles evolution in turbulent flames, which will be presented in Sec. 5 . 


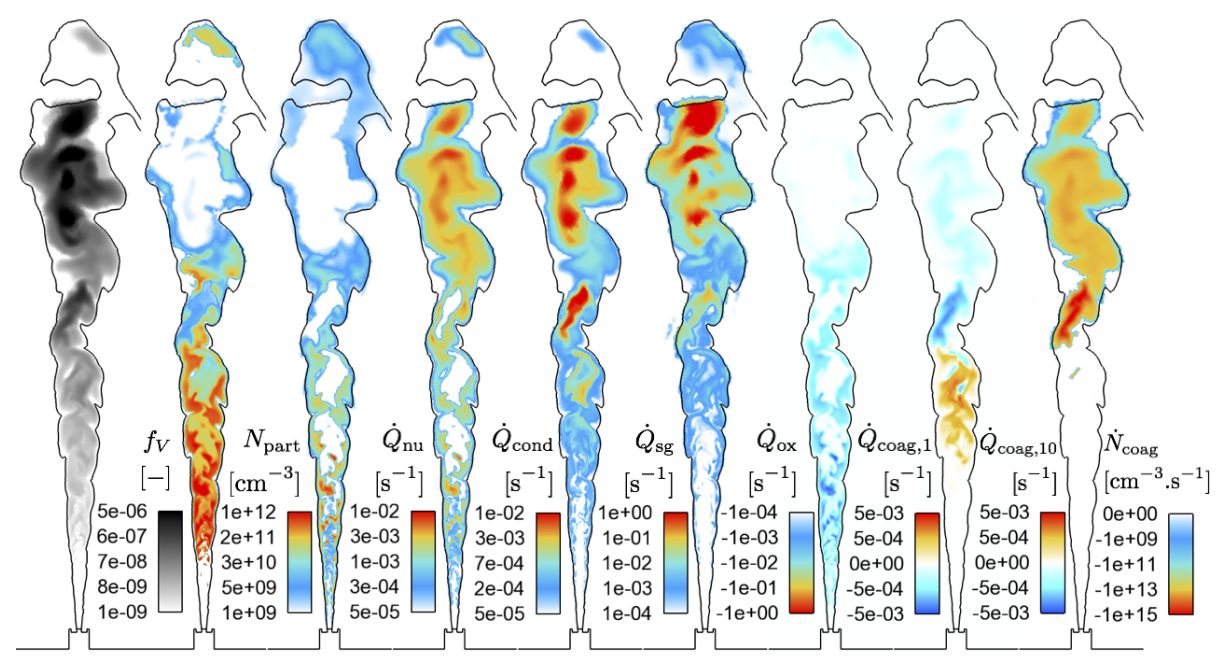

Figure 8. Instantaneous fields of soot volume fraction, particle number density, nucleation, condensation, surface growth and oxidation volume source terms for all the sections, volume coagulation source term for the first and tenth section and number coagulation source term for all the sections. The iso-contour of mixture fraction at $Z_{0}$ (indicating the flame front) is shown in solid line.

\section{Numerical characterization of the evolution of the soot production in a turbulent flame}

This section presents a characterization of soot production evolution in the studied non-premixed ethylene/air flame by analyzing all the information accessible thanks to the coupling of the LES approach and the sectional method.

\subsection{Global quantities}

Figure 8 shows instantaneous fields of soot volume fraction $f_{V}$ and of particles number density $N_{\text {part }}$. An iso-contour of mixture fraction at value $Z_{0}$ localizing the flame front is also shown with a solid line. It can be noticed that soot particles are always located on the rich side of the flame $\left(Z>Z_{0}\right)$.

In order to understand the phenomena governing the $N_{\text {part }}$ and $f_{V}$ fields, Fig. 8 also presents the instantaneous total soot volume fraction source terms: nucleation $\dot{Q}_{\mathrm{nu}}$, condensation $\dot{Q}_{\text {cond }}$, surface growth $\dot{Q}_{\mathrm{sg}}$ and oxidation $\dot{Q}_{\mathrm{ox}}$. 
Nucleation occurs at the bottom of the flame where PAHs concentrations are high due to very rich mixtures. Condensation occurs all over the flame, while surface growth and oxidation are more located downstream in the flame. It can be seen that maximum surface growth and oxidation source terms are at least two order of magnitudes higher than nucleation and condensation phenomena. Then, with this presented model and for the studied flame, surface reactivity source terms are the main contributors in soot volume fraction production. Surface growth occurs at the middle of the flame whereas oxidation is mainly present at the top of the flame, but also near the stoichiometric iso-contour where particles are oxidized. It is important to note that these observations are in contrast with previous studies on similar flames. In the studies presented in $[4,21]$, nucleation and condensation were found to be the major processes involved in soot production. However, in other studies [? 8], surface growth was found to be at least of the same order of magnitude as PAH-related soot growth pathways. In the current study, surface growth is identified to be the main process involved in soot particles growth. This variability in results can be due to the large diversity that exists between the different sub-models and constants used for each one of the soot formation processes in the different works. At the same time, the investigated flames were also different and today there is no proof that a specific hierarchy exists between the numerous phenomena governing soot production common to all turbulent flames.

Coagulation does not alter the total volume source term, but it may be interesting to look at the effect of coagulation in terms of section distribution. Therefore, the coagulation source terms are presented in Fig. 8 for the first $\left(\dot{Q}_{\text {coag, } 1}\right)$ and the tenth sections $\left(\dot{Q}_{\text {coag, } 10}\right)$ of the soot particle size distribution, corresponding respectively to particles with mean volumes $v_{1}^{\text {moy }}=0.9 \mathrm{~nm}^{3}$ and $v_{10}^{\text {moy }}=4 \cdot 10^{3} \mathrm{~nm}^{3}$. For the first section, soot coagulation source term is always negative because of the coagulation of the smallest particles to form bigger ones. For the tenth section, it is first positive because of the coagulation of soot particles from the smallest soot sections, then becomes negative because of the coagulation of the particles from the tenth section towards bigger soot sections. 
The coagulation source term of the total number density $\dot{N}_{\text {coag }}$ calculated as in Eq. (34) is also presented in Fig. 8. Comparing the fields of $\dot{N}_{\text {coag }}$ and $N_{\text {part }}$, one can observe that the decrease of particles number density at the middle of the flame is linked to coagulation leading to the presence of big soot particles. As a consequence, condensation becomes more important than nucleation in this region, where particle diameters are much larger than the dimer diameter so that condensation rate (collision of one soot particle with a dimer particle) is higher than nucleation (collision of two dimer particles).

In conclusion, in the present simulation we observe that the particle number density is higher at the bottom of the flame where nucleation process is important and decreases downstream due to the coagulation phenomenon. On the contrary, soot volume fraction increases along the axial position mainly due to surface growth and then decreases up to the flame where $f_{V}$ is oxidized due to the presence of $O H$ radical.

In order to confirm the tendencies observed on the instantaneous fields, Fig. 9 gives a representation of the localization of the total nucleation, condensation, surface growth and oxidation source terms. The colored region corresponds to the zone where the mean source term is higher than $25 \%$ of its maximum value. The location of the PAH, $\mathrm{C}_{2} \mathrm{H}_{2}$, and $\mathrm{OH}$ mass fractions are also indicated. Nucleation and condensation source terms are linked to the presence of PAHs, and condensation occurs higher in the flame than nucleation (Fig. 9a). Indeed, as explained previously, soot nucleation is less probable than condensation once the size of the soot particles and, consequently, the number of big particles is high enough.

Concerning surface growth (Fig. 9b) and oxidation (Fig. 9c) phenomena, they are, as expected, linked to the presence of $\mathrm{C}_{2} \mathrm{H}_{2}$ and $\mathrm{OH}$ species, respectively, and to the presence of big particles since these phenomena depend on the particle surface.

Figures 10 present the normalized mean fields of the soot coagulation source terms for several selected sections. For the first section (Fig. 10a), soot particles always coagulate by forming bigger particles so that the source term is 
negative everywhere. For sections 5 (Fig. 10b) and 10 (Fig. 10c), two regions can clearly be identified, corresponding to the coagulation of smaller particles to form particles in the corresponding section (in red) and the coagulation of the soot particles of the section towards bigger soot particles (in blue). For higher soot sections (Fig. 10d-e), the mean coagulation source term is positive so that coagulation from smaller soot sections predominates compared to coagulation towards bigger sections.

These first analyses illustrate the physical richness obtained through the proposed approach. Before exploring in details the results, it is important to identify where the subgrid soot model may affect the results. For this, the mean subgrid soot intermittency $\omega$ is presented in Fig. $10 \mathbf{f}$ ). When $\omega$ is near one, the subgrid model for the soot quantities is active, whereas when $\omega$ is closed 0 , the soot quantities are resolved on the grid and the effect of the soot subgrid model is negligible. It can be observed that starting from $x / D=50, \omega$ is lower than 0.1. As a consequence, the analysis will be performed in the following only for $x / D>50$, where results are expected to be only slightly affected by the soot subgrid model.

\subsection{Mean particle size distributions}

As already stated, the sectional method provides access to the PSD information that can be used to characterize particle formation and evolution along the flame. For this, Fig. 11 shows mean particle size distributions of soot particles at the centerline for different selected heights above the burner: $\left(\mathrm{d} N / \operatorname{d} \log \left(d_{a}\right)\right)_{i}=3 \ln (10) q_{i}$. For $x / D<75$, the PSD presents one peak. Downstream, more particles are found in higher soot sections and a two-peak distribution is observed. ${ }^{2}$ These results are qualitatively in agreement with the

\footnotetext{
${ }^{2}$ The particle size distribution is considered as a one-peak shape when the PSD is monotone, and as a two-peak shape elsewhere.
} 

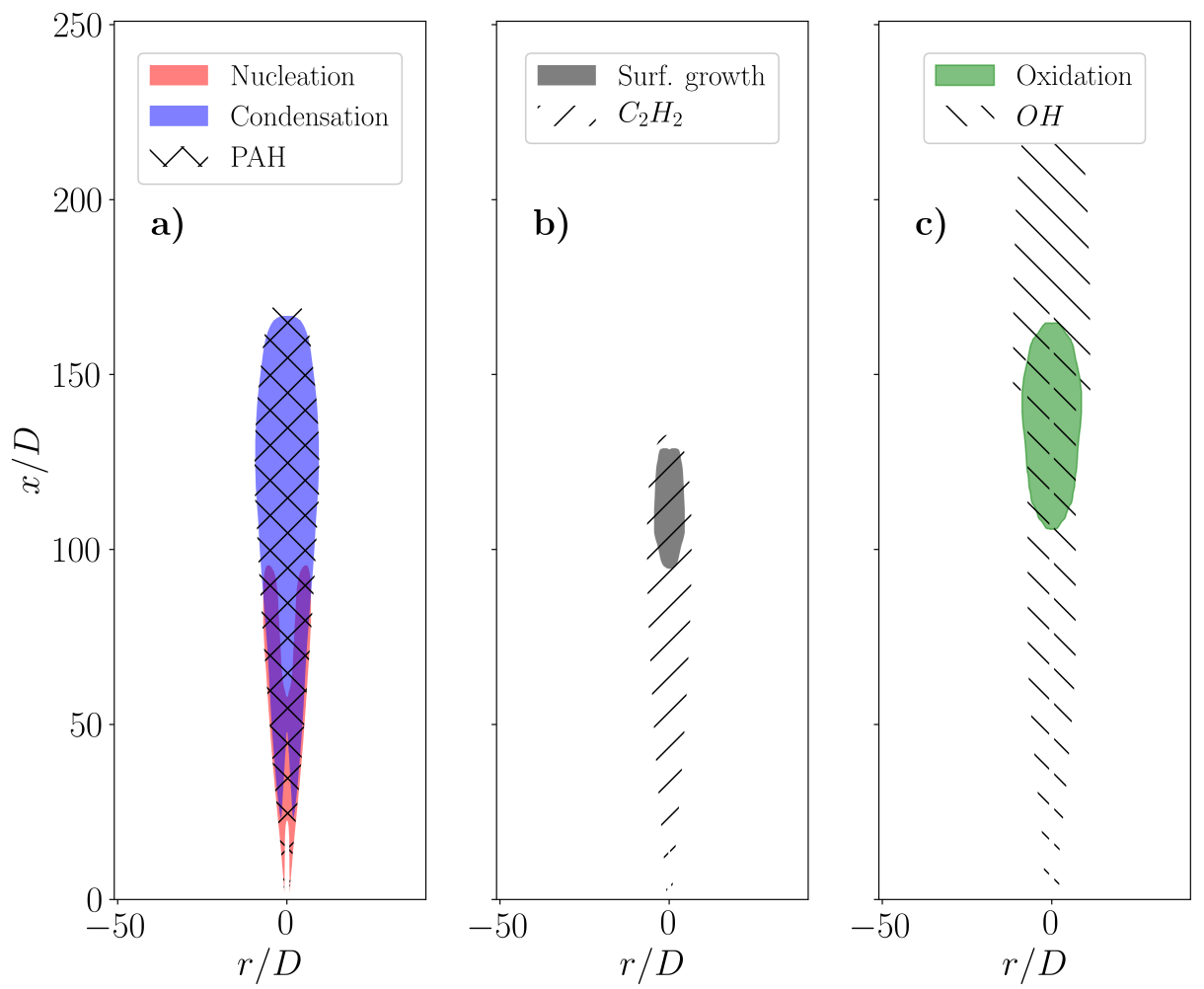

Figure 9. a) Nucleation and condensation presence indexes related to the presence of PAH precursors; b) Surface growth index related to the presence of $\mathrm{C}_{2} \mathrm{H}_{2}$ species; c) Oxidation index related to the presence of $\mathrm{OH}$ species 

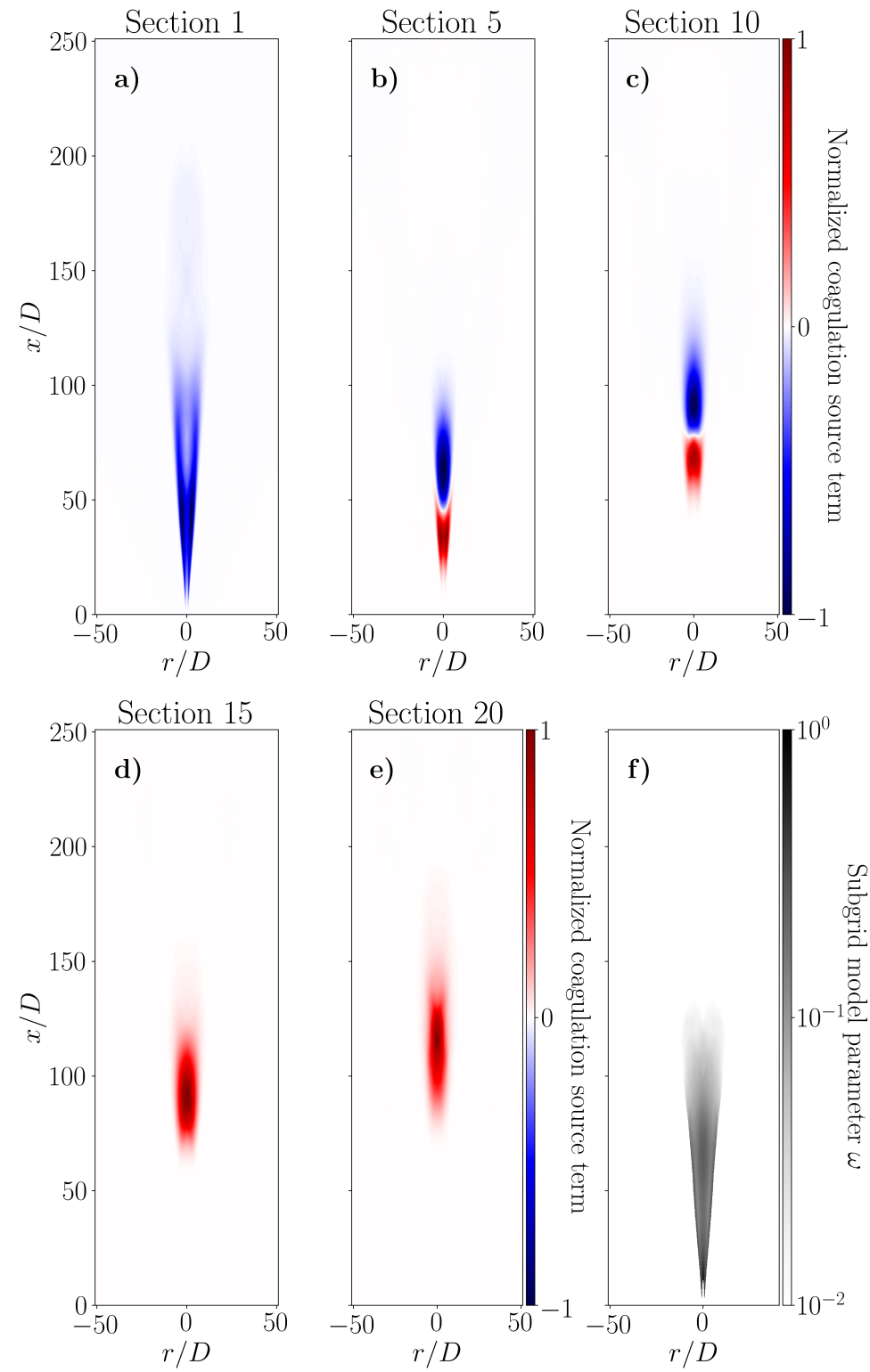

Figure 10. a)-e) Normalized mean fields of soot coagulation source terms for different soot sections; f) mean field of soot subgrid model parameter $\omega$ 
experimental tendencies only very recently observed in similar flames [? ? ].

Details of the different source terms involved for each section are shown in Fig. 12 for the same six heights.

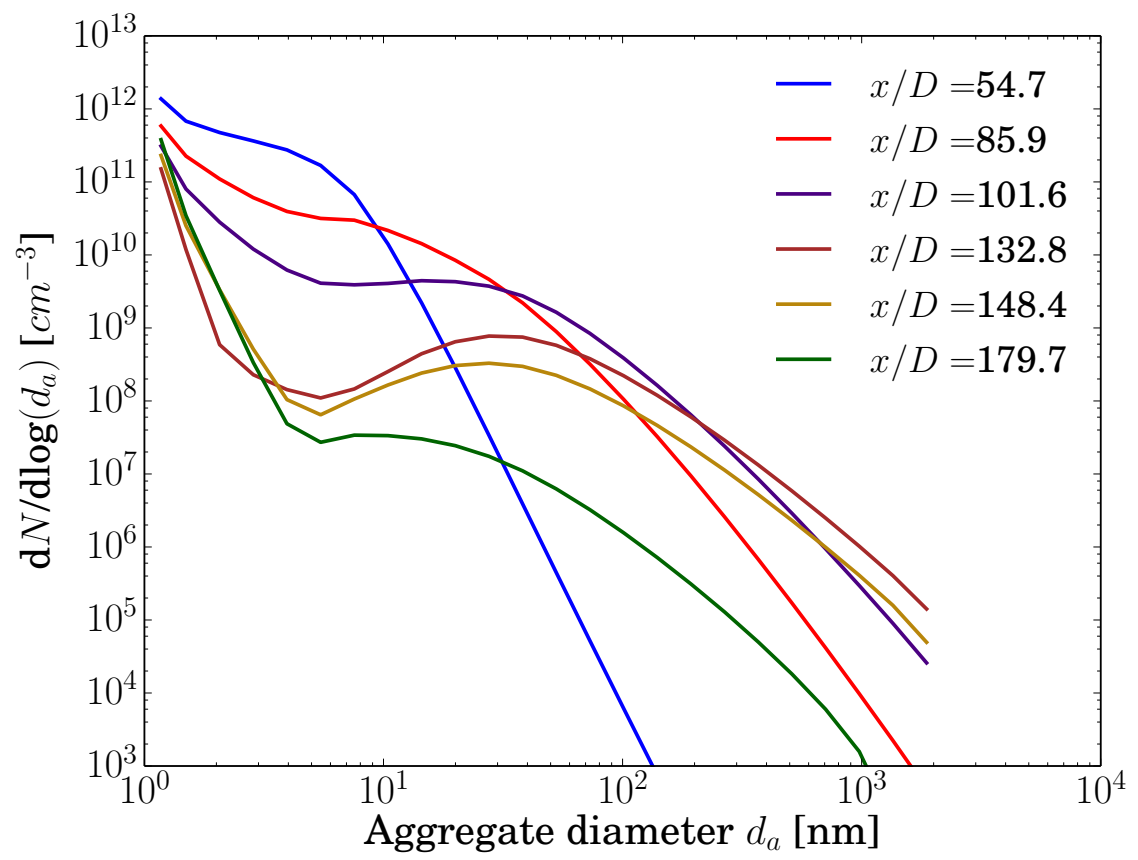

Figure 11. Mean particle size distribution of soot particles at the centerline for different selected heights above the burner.

For $x / D=54.7$, all the phenomena involved in soot production present almost the same order of magnitudes. Only small particles are present and, as mentioned before, the particle size distribution presents a one-peak shape corresponding to the nucleation of the smallest soot particles. At $x / D=85.9$, surface growth is the main phenomena involved in soot production. Oxidation and coagulation are also present and a two-peak shape of the PSD starts to be observed. The second peak of this two-peak PSD shape is more and more shifted toward bigger diameters mainly because of the growth and the coagulation of 
soot particles downstream in the flame. Then, between $x / D=101.6$ and $x / D=$ 148.4, a transition between the relative contributions of surface growth and oxidation is observed. Oxidation process is more and more important and at $x / D=179.7$, soot particles are totally oxidized.

Important physical processes information can then be captured with the presented approach enabling to understand the predicted mechanism of soot evolution in this flame. 

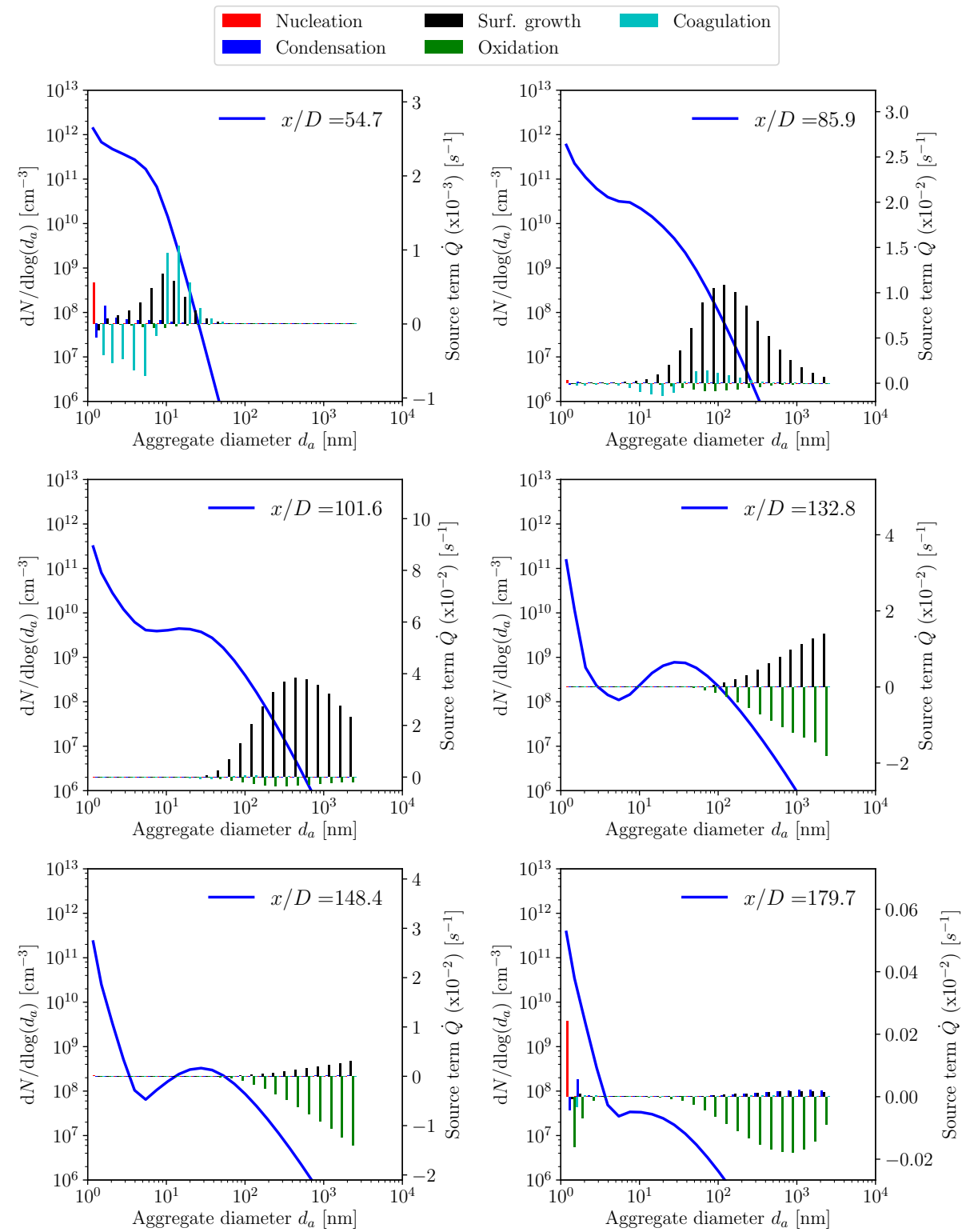

Figure 12. Mean particle size distributions and details of mean soot sections source terms at the jet centerline for different selected heights above the burner.

A representation of the mean soot particles size distribution shape along the 
flame is given in Fig. 13. Blue region corresponds to the zone where in average the PSD presents one peak whereas red region corresponds to the region where the PSD presents two peaks. Then, at the bottom of the flame, the PSD presents a one-peak shape whereas a two-peak PSD shape is observed downstream of the flame.

\subsection{Dynamic evolution of the PSD}

\subsubsection{PSD shape dynamics}

As mentioned in the introduction, the coupling of an LES approach with a sectional method provides unique information on the temporal evolution of the soot PSD in the flame. As an example, the temporal evolution of the PSD at $x / D=85.9$ (at the centerline) is presented in Fig. 14a showing high fluctuations with time from one-peak to two-peak shapes. 


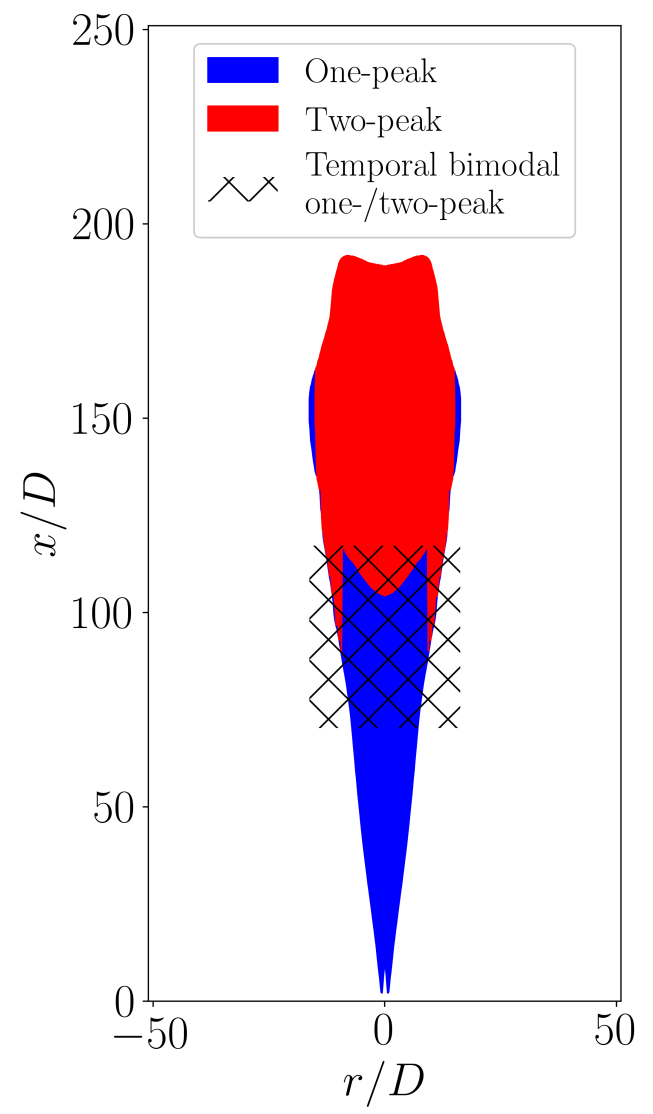

Figure 13. Index of the structure of the particle size distribution (one-peak, two-peak and temporal bimodality).

The same phenomena can be conveniently represented by looking at the probability density function (pdf) for each diameter, presented in Fig. 14b for the same position. The presented pdf is rescaled for each value of the aggregate diameter. Then, for each diameter, black points correspond to the most probable value of the particle size distribution function, whereas light grey points correspond to less probable values of the PSD. Looking at the pdf in Fig. 14b, the signature of a temporal bimodal behavior of the PSD is identified by the presence of two most probable values for small sections. 

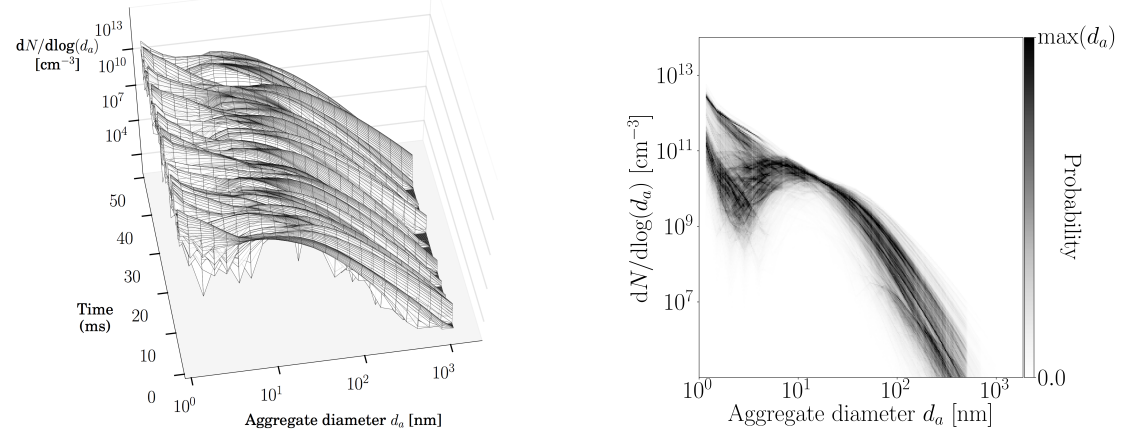

(a) Temporal evolution of the PSD for $x / D=(\mathbf{b})$ Probability density function of the PSD 85.9 for $x / D=85.9$

Figure 14. Unsteady evolution of particle size distribution of soot particles at the centerline for $x / D=85.9$.

In order to analyze the evolution of the structure of the PSD with time, Fig. 15 presents the probability density functions of particle size distributions for twelve different selected heights at the jet centerline:

- Important fluctuations of the first peak of the PSD are observed from $x / D=53.1$ to $x / D=62.5$ above the burner (Fig. 15a-d). However, there is no temporal bimodality and the PSD presents always a one-peak shape.

- From $x / D=70.3$ to $x / D=101.6$ (Fig. 15e-i), regular transitions between one-peak and two-peak PSD shapes can be observed. At $x / D=101.6$ (Fig. 15i), strong oscillations of the particle size distribution are observed. Soot volume fraction is the highest in the flame and the second peak of the particle size distribution is shifted towards big soot particles. The strong oscillations explain also the high RMS observed at these heights in Fig. 7. A very large spanning of PSD values is observed for big sections, increasing with the height above the burner.

- For the heights $x / D=132.8$ and $x / D=148.4$ (Fig. $15 \mathbf{j}-\mathbf{k}$ ), there is 
no temporal bimodality anymore. The PSD always presents a two-peak shape but the second peak is lower than in the previous heights because of the oxidation process that has already started. Compared to the second peak of the PSD, lower fluctuations of the first peak are observed. The spanning of values of the PSD increases until $x / D \approx 150$.

- At $x / D=179.7$ (Fig. 15l), as previously observed, big soot particles are almost totally oxidized. Only small soot particles remain at this height above the flame.

This analysis confirms the presence of a large region (presented in hatched in Fig. 13) where a temporal bimodal behavior is observed due to transition from a one-peak shape region located at the bottom of the flame and a two-peak shape zone downstream.

\subsubsection{Role of the particles history on the PSD}

In order to understand the origin of the PSD fluctuations, scatter plots of particle size distribution are plotted in Fig. 16 (left) colored by the value of the mixture fraction at three different heights.

It is first observed that depending on the position, the PSD does not have the same shape for a given value of the mixture fraction $Z$. However, it can be observed that at a fixed position in the temporal bimodal zone $(x / D=78.1$ and $x / D=101.6)$, the PSD tends to present a one-peak PSD shape in leaner pockets whereas a two-peak PSD shape is observed in richer pockets. This is confirmed by looking at the correspondent probability density functions of mixture fraction in Fig. 16 (right), which have been conditioned by the shape of the particle size distribution. It is again observed that two populations coexist for $70<x / D<110$ : a two-peak PSD shape in the richer pockets and a onepeak PSD shape in the leaner pockets. However, it can be concluded that the PSD-shape is not univocally governed by the mixture fraction and it seems that the particle history plays a role on the instantaneous PSD at a given flame point. 


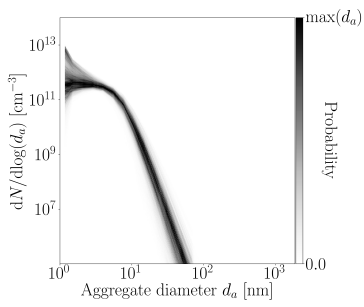

(a) $x / D=53.1$

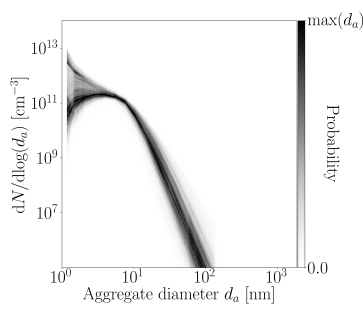

(d) $x / D=62.5$

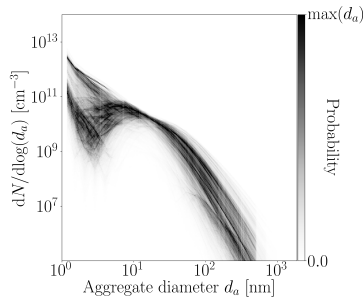

(g) $x / D=85.9$

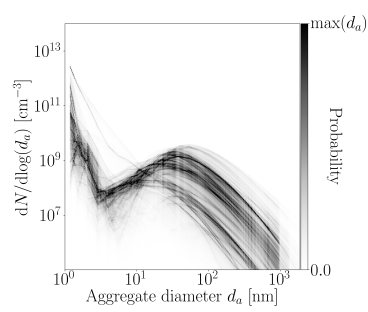

(j) $x / D=132.8$

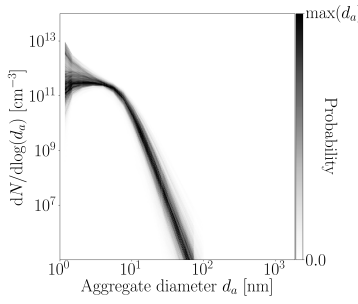

(b) $x / D=56.2$

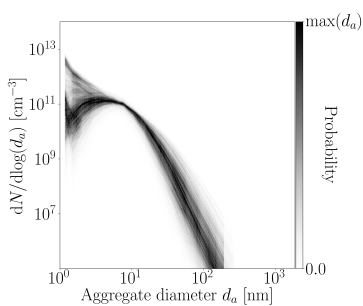

(e) $x / D=70.3$

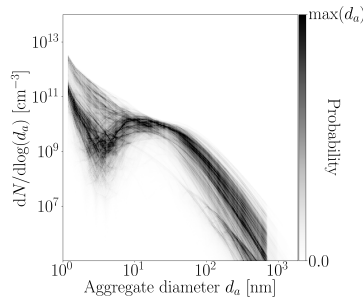

(h) $x / D=93.8$

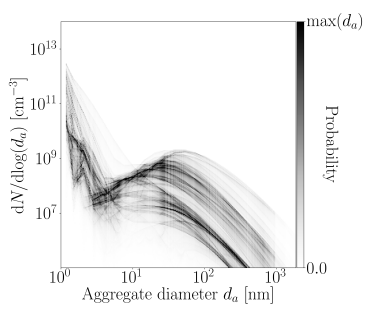

(k) $x / D=148.4$

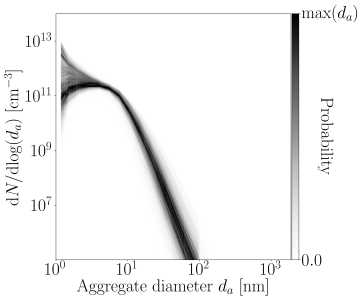

(c) $x / D=59.3$

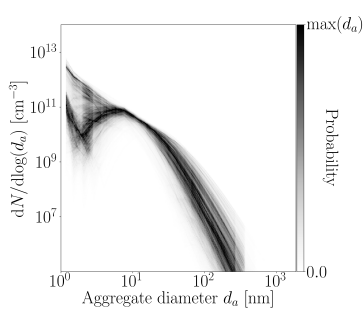

(f) $x / D=78.1$

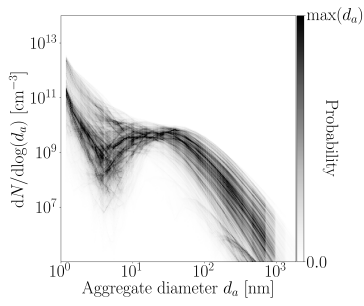

(i) $x / D=101.6$

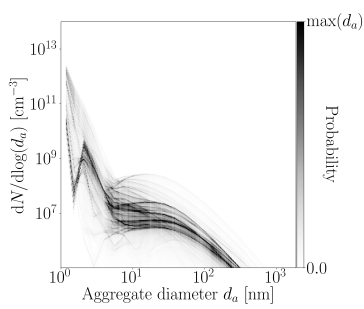

(l) $x / D=179.7$

Figure 15. Probability density functions of particle size distributions of soot particles at the centerline for twelve selected heights above the burner. 

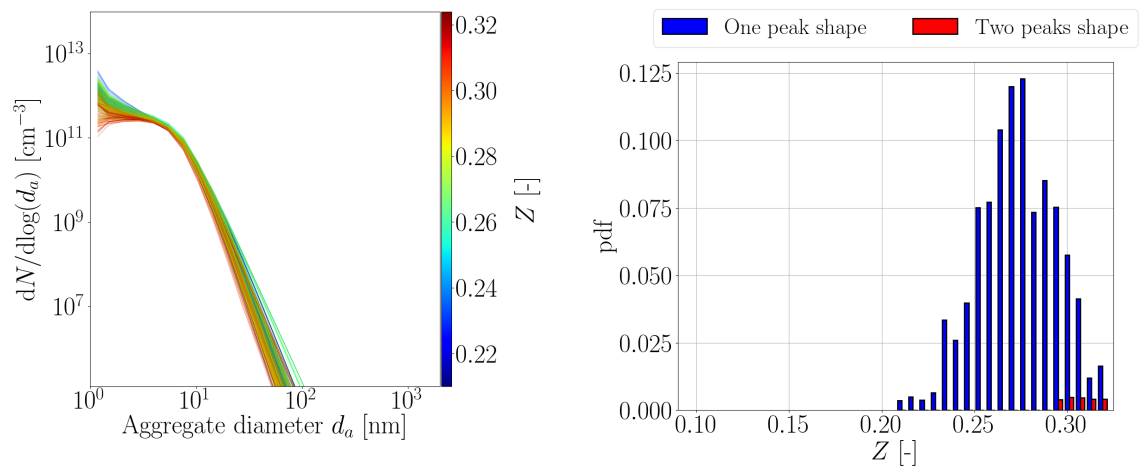

(a) $x / D=56.2$, i)

(b) $x / D=56.2$, ii)
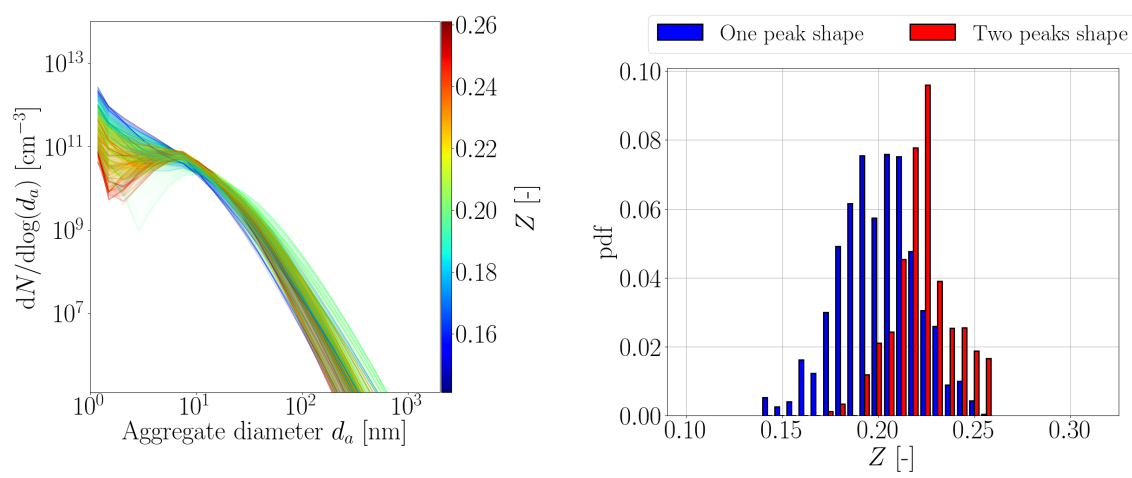

(c) $x / D=78.1$, i)

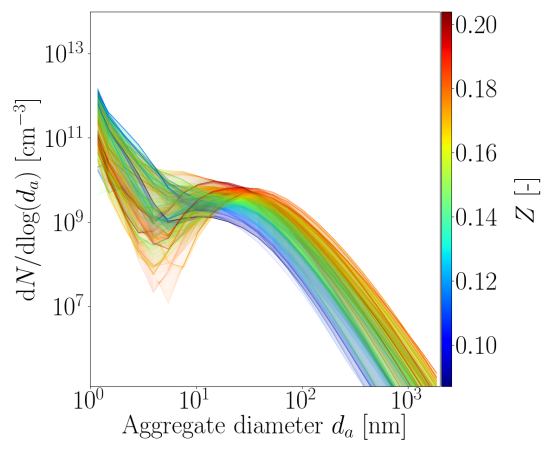

(d) $x / D=78.1$, ii)

(e) $x / D=101.6, \mathbf{i})$

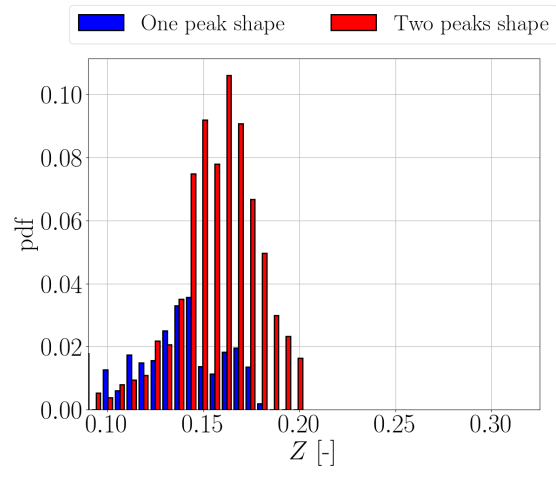

(f) $x / D=101.6$, ii)

Figure 16. For different heights above the burner:

i) left, Scatter plots of particle size distributions colored by the value of instantaneous mixture fraction,

ii) right, Probability density functions of the mixture fraction conditioned by the particle size distribution shape. 
In order to confirm it, a set of Eulerian instantaneous fields has been postprocessed in order to extract Lagrangian trajectories representative of soot particles. Two Lagrangian trajectories issued from the center of the ethylene tube exit have been extracted at two different instants for a total Lagrangian time of $14 \mathrm{~ms}$ each (Fig. 17). These trajectories have been computed taking into account both the fluid and the soot particles thermophoretic velocities, and can then be considered as tracers of small reactors moving at the velocity $\widetilde{\mathbf{u}}+\widetilde{\mathbf{v}_{\text {th }}}$. In this representation, turbulent transport and chemical/collisional source terms are then considered as source terms of the reactors. Their projected trajectories are represented together with an instantaneous field of soot volume fraction $f_{V}$ and the sets of data presented hereafter refer to the corresponding extracted Eulerian fields at each position and time for each Lagrangian trajectory. The first trajectory (solid purple line in Fig. 17a) ends up in a one-peak shape particle size distribution at $x / D=76.9 D$, Fig. $17 \mathbf{b}$, solid line. The second trajectory (dashed purple line in Fig. 17a) ends up in a two-peak shape particle size distribution (dashed line) at $x / D=92.8 \mathrm{D}$, Fig. $17 \mathbf{c}$, dashed line.

The corresponding source terms are plotted in Fig. 17b-c with the particle size distribution for the final Lagrangian time of the trajectory $\left(\tau_{f}=14 \mathrm{~ms}\right)$. Looking at the final values of the source terms, the two trajectories end with two different soot formation process steps. For the first trajectory, all the source terms have the same order of magnitude. Nucleation and condensation are still important processes of the soot particles evolution. For the second trajectory, nucleation and condensation are still active processes (even if they are not visible in Fig. 17c because of the difference of magnitudes between the different soot formation processes), but the main contributors of soot particles evolution at the final position are the surface reactivity processes (surface growth and oxidation). Their order of magnitude per section is one order of magnitude $\left(\approx 10^{-2} \mathrm{~s}^{-1}\right)$ higher than the ones obtain for the ones obtained for the first trajectory $\left(\approx 10^{-3}\right.$ $\left.\mathrm{s}^{-1}\right)$.

As we mentioned before, surface growth and oxidation are the main contributors of particles growth in this simulation. It was also noted that these 
phenomena are highly linked to the presence of $\mathrm{C}_{2} \mathrm{H}_{2}$ for surface growth and $\mathrm{OH}$ for oxidation processes (Fig. 9), so that such behavior is expected to be linked to the evolution of these quantities along the particle trajectories.

To verify it, the evolution of the Lagrangian temporal mean mass fraction of $C_{2} H_{2}$, i.e. $1 / \tau \int_{0}^{\tau} Y_{C_{2} H_{2}}\left(\tau^{\prime}\right) d \tau^{\prime}$ and Lagrangian temporal mean mass fraction of $O H$, i.e. $1 / \tau \int_{0}^{\tau} Y_{O H}\left(\tau^{\prime}\right) d \tau^{\prime}$ are represented respectively in Fig. $18 \mathbf{a}$ and Fig. 18b. The corresponding evolution of the mixture fraction along the trajectory is plotted in Fig. 18c. Finally, the Lagrangian time integral of the sum of the surface growth and oxidation source terms, i.e. $\int_{0}^{\tau}\left(\dot{Q}_{s g}\left(\tau^{\prime}\right)+\dot{Q}_{o x}\left(\tau^{\prime}\right) d \tau^{\prime}\right.$, is plotted in Fig. 18d. For all these figures, the solid lines correspond to the first trajectory resulting in a one-peak PSD shape, whereas the dashed lines correspond to the second trajectory resulting in a two-peak PSD shape. First, it can be observed that for $\mathrm{C}_{2} \mathrm{H}_{2}$ mass fraction, both trajectories follow quite the same evolution (Fig. 18a). However, at the end, the first particle trajectory will reach a leaner mixture region compared to the second trajectory (cf. Fig. 18c), where the $\mathrm{OH}$ mass fraction is high (cf. Fig. 18b). The resulting evolution of the Lagrangian time integral of the sum of the surface growth and oxidation source terms is then affected by these processes. Indeed, it can be seen in Fig. 18d, that an equilibrium between surface growth and oxidation processes is observed for the first trajectory (in solid line), whereas for the second one, surface growth process evolution increases faster than the oxidation process, enabling particles to grow and therefore to have a higher rate of coagulation. In this case, as surface growth boosts the coagulation of soot particles and nucleation is still an active process, particles are finally distributed in a two-peak PSD shape.

The particle size distribution is then strongly affected by the local gaseous conditions encountered by the particles along their trajectories.

Two other Lagrangian trajectories are studied in Fig.19. Both are issued from the fuel injector and their projected trajectories are plotted in Fig. 19a. However, the first trajectory (in solid lines) ends up with a one-peak PSD shape 

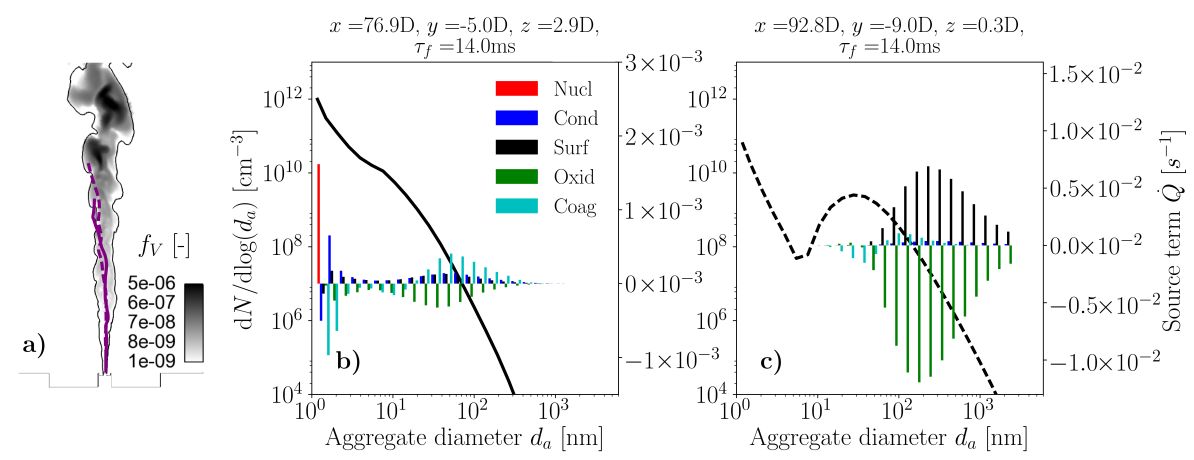

Figure 17. Final particle size distributions (lines) and final soot sectional source terms (bars) of two Lagrangian trajectories issued from the center of the jet and with a total Lagrangian time of 14 ms resulting in a one-peak (b)) and two-peak (c)) PSD shapes. The projected trajectories (in solid purple line for the the trajectory resulting in a one-peak shape and in dashed line for the trajectory resulting in a two-peak shape) are represented with an instantaneous field of soot volume fraction $f_{V}(\mathbf{a})$ ).

with a total integrated Lagrangian time $\tau_{f}=10.7 \mathrm{~ms}$, whereas the second one (in dashed lines) ends up in a two-peak PSD shape with a total integrated Lagrangian time $\tau_{f}=12.4 \mathrm{~ms}$. Here, the total Lagrangian time is different for the two trajectories but the final position coincides $(x / D=78.1)$. One trajectory results in a one-peak PSD shape (Fig. 19b) and the other one in a two-peak PSD shape (Fig. 19c). Their corresponding final soot sectional source terms are also plotted in Fig. 19b-c (bars). For both trajectories, at the end, surface growth and coagulation are the main contributors of soot particles evolution. The same order of magnitudes for the final source terms are obtained for both trajectories. However, as the total integrated Lagrangian time $\tau_{f}$ of the second trajectory is higher than the first one, the particles following the second trajectories spend more time in pockets where surface growth is active so that a two-peak PSD is finally observed. This analysis is confirmed by looking at the evolution of the Lagrangian time integral of the sum of the surface growth and oxidation source terms (Fig. 20d), which is linked to the evolution of the Lagrangian temporal mean mass fraction of $\mathrm{C}_{2} \mathrm{H}_{2}$ (Fig. 20a), of the Lagrangian temporal mean mass fraction of $O H$ (Fig. 20b) and of the mixture fraction (Fig. 

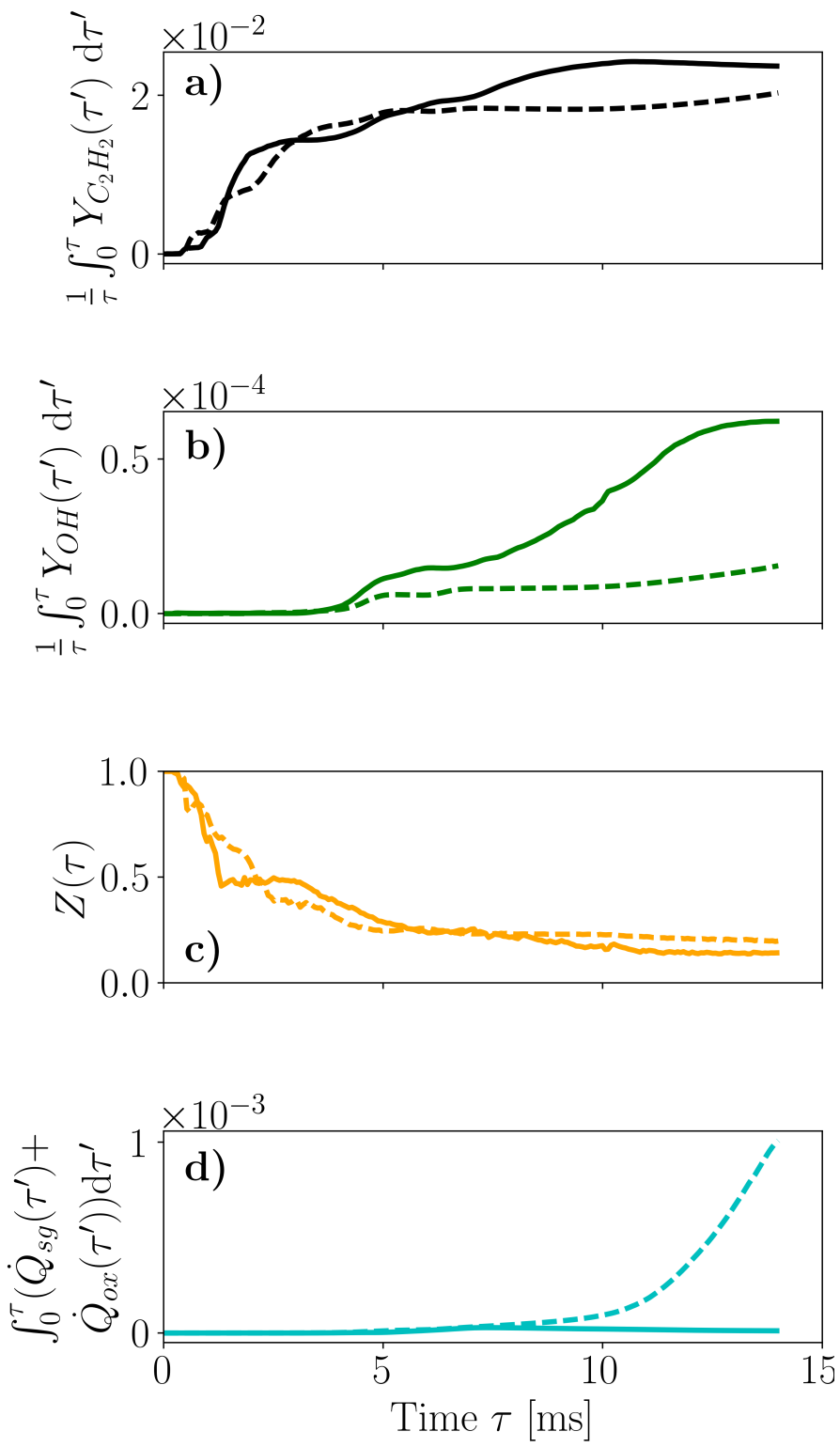

Figure 18. Lagrangian temporal mean mass fraction of $\mathrm{C}_{2} \mathrm{H}_{2}$, Lagrangian temporal mean mass fraction of $O H$, mixture fraction $Z$, and Lagrangian time integral of the sum of the surface growth and oxidation source terms evolutions for the trajectories of Fig. 17 resulting in a one-peak PSD shape (solid lines) and in a two-peak PSD shape. 
20c). This quantity evolves in the same way for both trajectories, but as the first trajectory Lagrangian time is shorter than that of the second trajectory, the total accumulated soot mass is lower than the second one, resulting then in a one-peak shape PSD. The total integrated path time of soot history plays also a role in the evolution of the PSD at a fixed position in the flame.
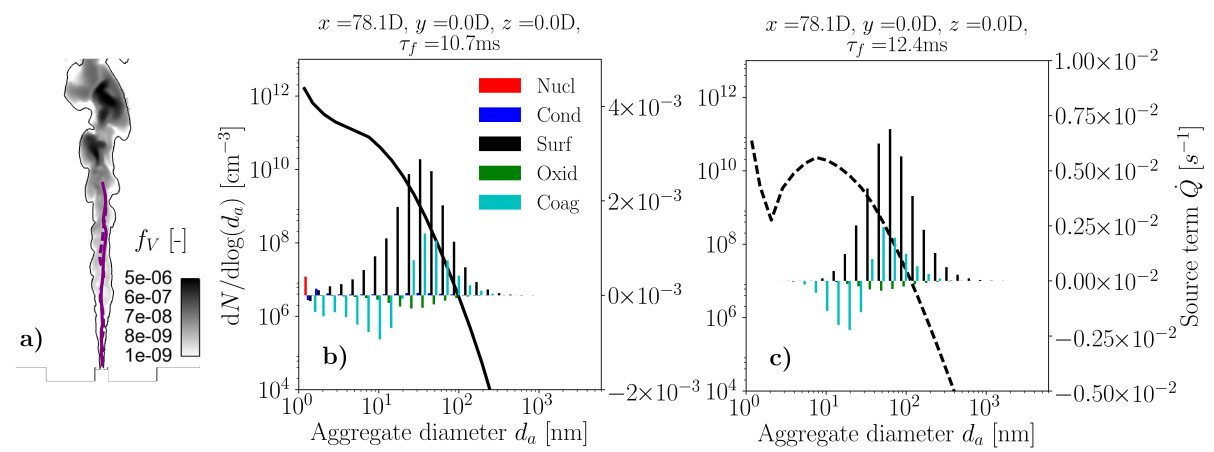

Figure 19. Projected trajectories, in solid purple line for the the trajectory resulting in a onepeak shape and in dashed line for the trajectory resulting in a two-peak shape, are represented with an instantaneous field of soot volume fraction $f_{V}(\mathbf{a})$. Final particle size distributions (lines) and final soot sectional source terms (bars) of two Lagrangian trajectories issued from the jet and resulting in a one-peak (b) and two-peak (c) PSD shapes at the same position.

Overall, it can be concluded that the particles history, i.e. the succession of the chemical and collisional phenomena experienced by the soot population along its trajectory in the flame (mainly driven by turbulence), is responsible for the high temporal fluctuations observed in the PSD shapes.

\section{Interpretation of soot presence indexes}

Having access to full information on soot quantities in the numerical simulations, it may be interesting to interpret the experimental soot intermittency index in light of the numerical observations. Due to experimental constraints, this quantity is measured by fixing a threshold $\epsilon_{f_{V}}$ (chosen in [63] as $\epsilon_{f_{V}}=0.03$ ppm), so that if $f_{V}$ is lower than $\epsilon_{f_{V}}$, it is considered that no soot is observed. Therefore, the soot intermittency index, indicating the probability of having $(0)$ 

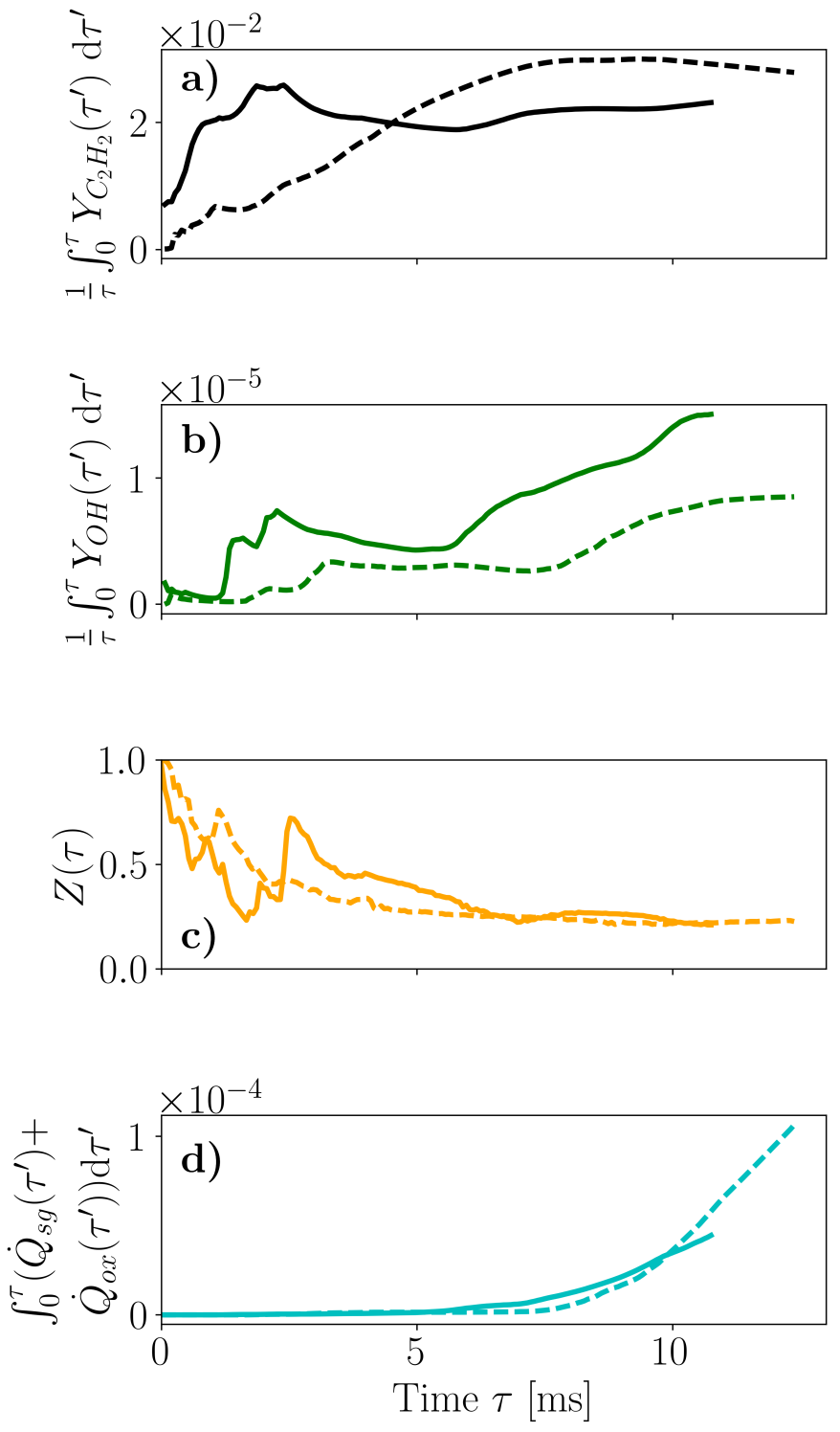

Figure 20. Lagrangian temporal mean mass fraction of $\mathrm{C}_{2} \mathrm{H}_{2}$, Lagrangian temporal mean mass fraction of $O H$, mixture fraction $Z$, and Lagrangian time integral of the sum of the surface growth and oxidation source terms evolutions for the trajectories of Fig. 19 resulting in a one-peak PSD shape (solid lines) and in a two-peak PSD shape (dashed lines). 
or not having (1) soot, may be affected by the value of $\epsilon_{f_{V}}$. In order to investigate this point, the numerical mean soot index is plotted in Fig. 21, while retaining the same threshold. At the bottom of the flame, a very low probability of having soot particles is observed, while high probability is obtained between $x / D=75$ and $x / D=175$.

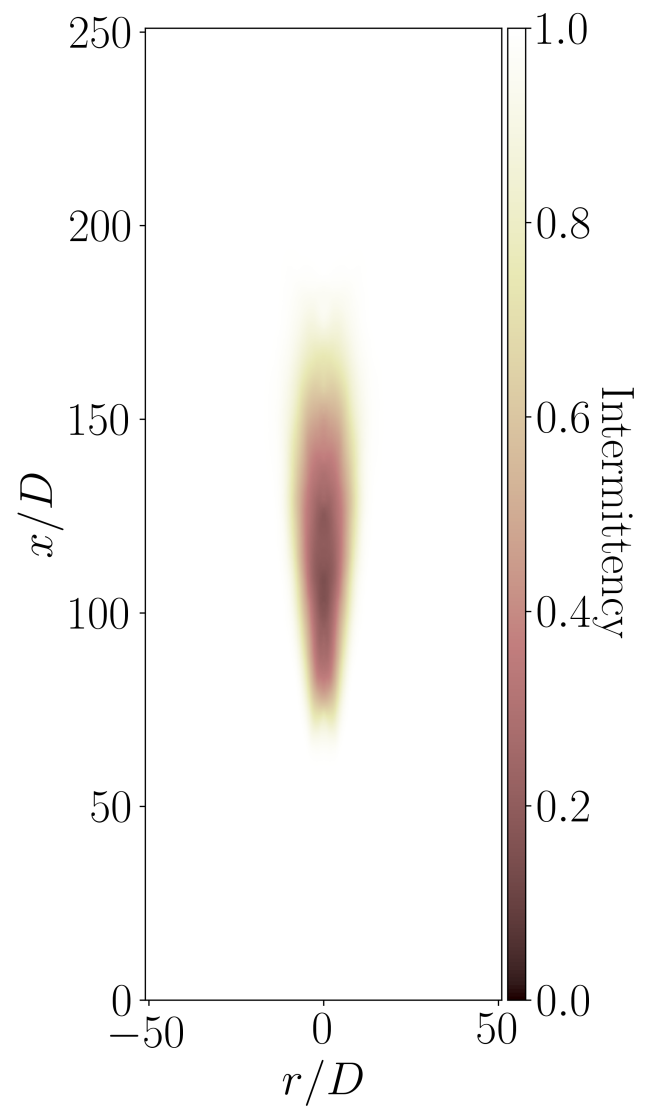

Figure 21. Mean field of resolved temporal soot intermittency.

In addition, Fig. 22 presents the temporal evolution of the PSD shape for two selected heights in the transition region $(x / D=78.1$ and $x / D=93.8)$. A value of one corresponds to a two-peak PSD shape whereas a value of zero 
corresponds to a one-peak PSD shape. Grey regions correspond to instants where the classical soot intermittency index i.e. $f_{V}>0.03 \mathrm{ppm}$ indicates that soot particles are present. High intermittency is observed in soot presence and two-peak PSD shape signals. These results point out the existence of a strong correlation for this flame between the two-peak PSD shape criterion and this definition of soot intermittency index, i.e. that for this specific threshold only two-peak populations of soot are detected.

In order to confirm it, a two-peak PSD shape index is evaluated as the probability of having (0) or not having (1) a two-peak PSD (Fig. 23) and compared with the experimental and numerical results for the classical definition of soot intermittency. As previously observed, a relationship between soot presence and two-peak PSD shape is verified. This can be easily explained by the fact that in the investigated flow configuration, it has been observed that when the simulated soot volume fraction is above the experimental threshold (0.03 ppm), the PSD generally presents a two-peak shape, whereas a one-peak PSD shape is generally obtained for low values of soot volume fraction $\left(f_{V}\right)$, below the experimental threshold.

In addition, a new index based on the probability of having the particles number density below an arbitrary threshold of $4 \cdot 10^{11} \mathrm{~cm}^{-3}$ has been calculated and is presented in Fig. 23. This definition is representative of the zones where soot particles are numerous and is not related to the mass of soot particles. It can be observed that this indicator does not show the same soot presence zones as the other ones. Indeed, with the classical definition of global soot intermittency, no soot presence is detected below $x / D=60$ while the indicator based on $N_{\text {part }}$ clearly shows a zone where a lot of (small) soot particles are present.

Therefore, due to the experimental threshold, the soot intermittency identifies regions where soot volume fraction is high enough to be measured, and, for this specific configuration, is strongly correlated with presence of a two-peak 


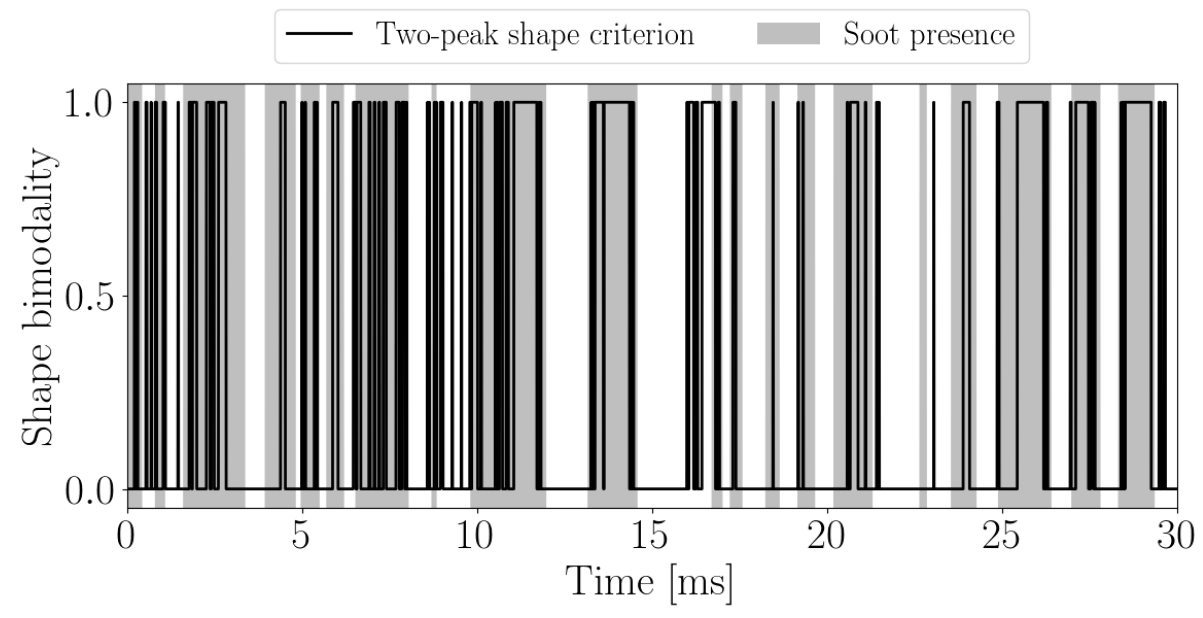

(a) $x / D=78.1$

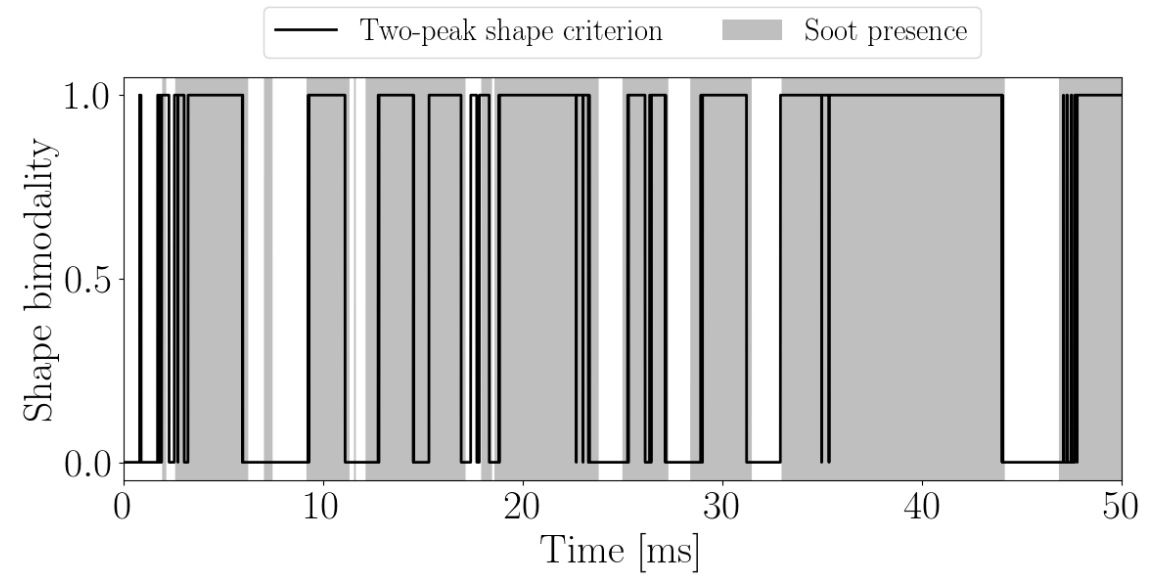

(b) $x / D=93.8$

Figure 22. Time evolution of shape bimodality criterion for two selected heights above the burner. A value of 1 corresponds to a two-peak shape, whereas 0 corresponds to a one-peak shape. Soot presence index is indicated in grey filled regions.

PSD. However, it is important to note that this observation may vary depending on the studied configuration, and that no causality between two-peak PSD and soot intermittency can be stated. 


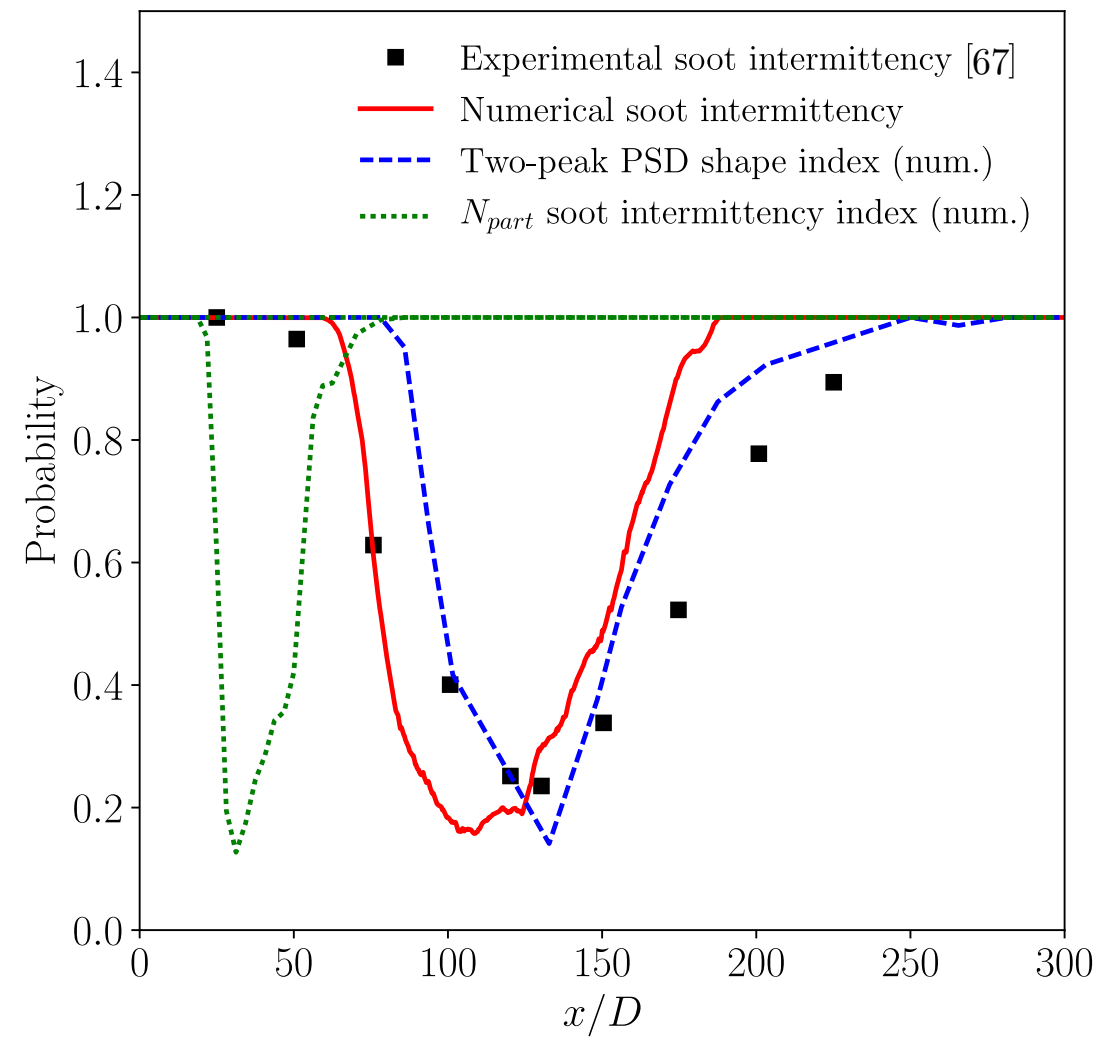

Figure 23. Comparison between experimental intermittency index, numerical soot intermittency index, two-peak PSD shape index and soot intermittency index based on $N_{\text {part }}$.

\section{Conclusion}

A Large Eddy Simulation approach has been developed for sooting turbulent non-premixed flames.

It is based on:

- a soot sectional description previously validated in laminar premixed and diffusion flames,

- the Radiation Flamelet/Progress Variable for turbulent combustion model including heat losses due to gas phase and soot particles radiation, 
- a relaxation model for PAHs evolution description enabling to capture the slow chemistry of soot precursors evolution,

- a soot subgrid model based on a presumed subfilter PDF approach previously developed for the hybrid method of moments, adapted to the sectional model.

The model has then been applied to the simulation of an experimental jet ethylene-air diffusion flame. A good agreement with experiments is obtained between numerical profiles of mean and RMS temperature and non-quantitative $\mathrm{OH}$ profiles compared. Concerning soot evolution, good predictions of soot position and soot volume fraction are obtained. Good dynamics of soot evolution production are also achieved. Indeed, soot intermittency is well predicted compared to experiments, which allows to be confident of the soot particles dynamics description.

Once validated, the numerical results have been investigated by analyzing the soot source terms. Surface reactivity of soot particles has been identified as the most important contributions to the total soot mass production and destruction. These observations differ with previous studies. Indeed, depending on the model used to describe the solid phase, previous studies $[4,21]$ found that PAH-related soot growth pathways were the major contributor of soot production in this kind of flames but, others [? 8] found that surface and PAH-related growth pathways were responsible in a similar way of soot particles growth. Further fundamental studies are therefore still necessary. However, it is impossible today to say if it really exists a hierarchy among the different processes for all turbulent flames and which is the model capable to reproduce it.

In addition, to characterize soot production in turbulent flames:

- Soot particles size distributions have been analyzed thanks to the validated sectional model. A one-peak PSD shape is first observed for $x / D<70$ whereas a two-peak PSD shape is observed higher in the flame. Once the two-peak shape is obtained and before total oxidation at $x / D \approx 180$, 
the second peak of the PSD distribution is shifted to bigger soot particles along the flame.

- The temporal evolution of the PSD has been characterized at different positions of the flame. It has been shown that it is subjected to strong fluctuations, whose spanning increases with the height above the burner. In addition, a temporal bimodal region is identified at $70<x / D<110$, where both one-peak and two-peak PSD shapes can be observed. By analyzing Lagrangian trajectories, it has been shown that soot history, i.e. the succession of the chemical and collisional phenomena experienced by the soot population along the flame, is responsible for such bimodal behavior.

- Finally, the role of the experimental threshold for the definition of the intermittency index has been investigated. It has been shown that for this configuration, the intermittency index, which is linked to the zones where $f_{V}$ is high, mainly localizes the presence of a two-peak PSD shape, while it neglects the presence of small particles, generally correlated with the particle number density $N_{\text {part }}$.

In conclusion, by coupling Large Eddy Simulations and sectional approach for soot particles description, more detailed information about soot particles population and their dynamics have been obtained. History evolution of soot

particles can be explained and the major contributors of soot evolution can be identified.

\section{Acknowledgments}

This study is supported by the Air Liquide, CentraleSupelec and CNRS Chair on oxycombustion and heat transfer for energy and environment and by the OXYTEC project, grant ANR-12-CHIN-0001 of the French Agence Nationale de la Recherche. It was also granted access to the HPC resources of CINES, IDRIS and TGCC under the allocation 2016-020164 made by GENCI. 


\section{References}

[1] J. Quaas, Global warming: The soot factor, Nature 471 (7339), (2011) $456-457$.

[2] J. S. Lighty, J. M. Veranth, A. F. Sarofim, Combustion aerosols: factors governing their size and composition and implications to human health., J. Air Waste Manag. Assoc. 50 (9), (2000) 1565-1618.

[3] Y. Xin, J. P. Gore, Two-dimensional soot distributions in buoyant turbulent fires, Proc. Combust. Inst. 30 (1), (2005) 719-726.

[4] F. Bisetti, G. Blanquart, M. E. Mueller, H. Pitsch, On the formation and early evolution of soot in turbulent nonpremixed flames, Combust. Flame 159 (1), (2012) 317-335.

[5] A. Attili, F. Bisetti, M. E. Mueller, H. Pitsch, Formation, growth, and transport of soot in a three-dimensional turbulent non-premixed jet flame, Combust. Flame 161 (7), (2014) 1849-1865.

[6] D.O. Lignell, J.H. Chen, P.J. Smith, Three-dimensional direct numerical simulation of soot formation and transport in a temporally evolving nonpremixed ethylene jet flame, Combust. Flame 155 (1-2), (2008) 316-333.

[7] P.G. Arias, V.R. Lecouster, Z. Luo, D.C. Haworth, T. Lu, A. Trouvé, H.G. Im, Dynamics of flow-soot interaction in wrinkled non-premixed ethylene-air flames, Combust. Theor. Model. 19 (5), (2015) 568-586.

[8] H. El-Asrag and S. Menon, Large eddy simulation of soot formation in a turbulent non-premixed jet flame, Combust. Flame 156 (2), (2009) 385395.

[9] M.E. Mueller and H. Pitsch, Large eddy simulation of soot evolution in an aircraft combustor, Phys. Fluids 25 (11), (2013) 1-20. 
[10] B. Franzelli, E. Riber, B. Cuenot, M. Ihme, Numerical Modeling of Soot Production in Aero-Engine Combustors Using Large Eddy Simulations. , Proceedings of GT2015, ASME Turbo Expo 2015, GT2015-43630, (2015).

[11] C. Eberle, P. Gerlinger, K.P. Geigle, M. Aigner, Numerical Investigation of Transient Soot Evolution Processes in an Aero-Engine Model Combustor, Combust. Sci. Technol. 187 (12), (2015) 1841-1866.

[12] H. Koo, M. Hassanaly, V. Raman, M.E. Mueller , K.P. Geigle, Large-Eddy Simulation of Soot Formation in a Model Gas Turbine Combustor, ASME. J. Eng. Gas Turbines Power. 139 (3), (2016) 031503-031503-9.

[13] K. Leung, R. Lindstedt, W. Jones, A simplified reaction mechanism for soot formation in nonpremixed flames, Combust. Flame 87 (3-4), (1991) $289-305$.

[14] R.P. Lindstedt, A Simple Reaction Mechanism for Soot Formation in NonPremixed Flames, Aerothermodynamics in Combustors (1992) 145-156.

[15] M. Frenklach and H. Wang, Detailed mechanism and modeling of soot particle formation, Soot Formation in Combustion, H. Bockhorn (Ed.), (1994) 162-190.

[16] G. Blanquart and H. Pitsch, A joint volume-surface-hydrogen multi-variate model for soot formation, Combustion Generated Fine Carbonaceous Particles, H. Bockhorn, A. D'Anna, A.F. Sarofim, H. Wang (Eds.), (2007) 437-463.

[17] S. Salenbauch, A. Cuoci, A. Frassoldati, C. Saggese, T. Faravelli, C. Hasse, Modeling soot formation in premixed flames using an extended conditional quadrature method of moments, Combust. Flame 162 (6), (2015) $2529-2543$.

[18] A. Wick, T-T. Nguyen, F. Laurent, R.O. Fox, H. Pitsch, Modeling soot oxidation with the Extended Quadrature Method of Moments, Proc. Combust. Inst. (36) (1), (2017) 789-797. 
[19] Y. Xuan, G. Blanquart, A flamelet-based a priori analysis on the chemistry tabulation of polycyclic aromatic hydrocarbons in non-premixed flames, Combust. Flame 161 (6), (2014) 1516-1525.

[20] A. Attili, F. Bisetti, M. E. Mueller, H. Pitsch, Effects of non-unity lewis number of gas-phase species in turbulent nonpremixed sooting flames, Combust. Flame (166), (2016) pp. 192-202.

[21] M. E. Mueller, H. Pitsch, LES model for sooting turbulent nonpremixed flames, Combust. Flame 159 (6), (2012) 2166-2180.

[22] Y. Xuan, G. Blanquart, Effects of aromatic chemistry-turbulence interactions on soot formation in a turbulent non-premixed flame, Proc. Combust. Inst. 35 (2), (2015) 1911-1919.

[23] M. Mueller, G. Blanquart, H. Pitsch, Hybrid method of moments for modeling soot formation and growth, Combust. Flame 156 (6), (2009) 1143 1155.

[24] J. L. Consalvi, F. Nmira, Transported scalar pdf modeling of oxygenenriched turbulent jet diffusion flames: Soot production and radiative heat transfer, Fuel 178, (2016) 37-48.

[25] P. Donde, V. Raman, M. E. Mueller, H. Pitsch, LES/PDF based modeling of soot-turbulence interactions in turbulent flames, Proc. Combust. Inst. 34 (1), (2013) 1183-1192.

[26] K. Netzell, H. Lehtiniemi, F. Mauss, Calculating the soot particle size distribution function in turbulent diffusion flames using a sectional method, Proc. Combust. Inst. 31 (1), (2007) 667-674.

[27] V. Fraioli, C. Beatrice, M. Lazzaro, Soot particle size modelling in 3-D simulations of diesel engine combustion, Combust. Theor. Model. 15 (6), (2011) 863-892. 
[28] D. Aubagnac-Karkar, J.-B. Michel, O. Colin, P. E. Vervisch-Kljakic, N. Darabiha, Sectional soot model coupled to tabulated chemistry for diesel RANS simulations, Combust. Flame 162 (8), (2015) 3081-3099.

[29] M. E. Mueller, H. Pitsch, Large eddy simulation subfilter modeling of sootturbulence interactions, Phys. Fluids (1994-present) 23 (11), (2011).

[30] W. Boyette, S. Chowdhury, W. Roberts Soot Particle Size Distribution Functions in a Turbulent Non-Premixed Ethylene-Nitrogen Flame, Flow Turbul. Combust. 98 (4), (2017) 1173-1186.

[31] S. Chowdhury, W. Boyette, W. Roberts Time-averaged probability density functions of soot nanoparticles along the centerline of a piloted turbulent diffusion flame using a scanning mobility particle sizer, J. Aerosol Sci. 106, (2017) 56-67.

[32] K. Netzell, H. Lehtiniemi, F. Mauss, Calculating the soot particle size distribution function in turbulent diffusion flames using a sectional method, Proc. Combust. Inst. 31 (1), (2007) $667-674$.

[33] C. Marchal, Modélisation de la formation et de l'oxydation des suies dans un moteur automobile, Ph.D. thesis, Université d'Orléans (2008).

[34] E. Cunningham, On the Velocity of Steady Fall of Spherical Particles through Fluid Medium, Proceedings of the Royal Society of London A: Mathematical, Physical and Engineering Sciences, 83 (563), (1910) 357365.

[35] P. Rodrigues, B. Franzelli, R. Vicquelin, O. Gicquel, N. Darabiha, Unsteady dynamics of PAH and soot particles in laminar counterflow diffusion flames, Proc. Combust. Inst. 36 (1), (2017) 927-934.

[36] B. V. Derjaguin, A. I. Storozhilova, Y. I. Rabinovich, Experimental verification of the theory of thermophoresis of aerosol particles, J. Colloid Interface Sci. 21 (1), (1966) 35-58. 
[37] M. E. Mueller, G. Blanquart, H. Pitsch, A joint volume-surface model of soot aggregation with the method of moments, Proc. Combust. Inst. $32(1),(2009) 785-792$.

[38] M. Smoluchowski, Versuch einer mathematischen Theorie der Koagulationskinetik kolloider Lösungen, 1916.

[39] M. Frenklach, On surface growth mechanism of soot particles, Symposium (International) on Combustion 26 (2), (1996) 2285 - 2293.

[40] F. Mauss, T. Schäfer, H. Bockhorn, Inception and growth of soot particles in dependence on the surrounding gas phase, Combust. Flame 99 (3-4), (1994) $697-705$.

[41] A. El-Leathy, F. Xu, G. Faeth, Soot surface growth and oxidation in laminar unsaturated-hydrocarbon/air diffusion flames, AIAA Journal, 2002.

[42] F. Xu, A. M. El-Leathy, C. H. Kim, G. M. Faeth, Soot surface oxidation in hydrocarbon/air diffusion flames at atmospheric pressure, Combust. Flame 132 (1-2), (2003) 43-57.

[43] D. Veynante and L. Vervisch, Turbulent combustion modeling, Prog. Energy Combust. Sci. 28 (3), (2002) 193-266.

[44] T. Poinsot and D. Veynante, Theoretical and numerical combustion, Third Edition, (2012).

[45] P. Moin, K. Squires, W. Cabot, S. Lee, A dynamic subgrid-scale model for compressible turbulence and scalar transport, Phys. Fluids A: Fluid Dynamics 3 (11), (1991) 2746-2757.

[46] B. Shotorban and S. Balachandar, A Eulerian model for large-eddy simulation of concentration of particles with small Stokes numbers, Phys. Fluids 19 (11), (2007) 118107.

[47] P. Sagaut Large Eddy Simulation for Incompressible Flows: An Introduction, Third Edition, Springer-Verlag Berlin Heidelberg, (2005). 
[48] F. Nicoud, F. Ducros, Subgrid-scale stress modelling based on the square of the velocity gradient tensor Flow Turbul. Combust. 62 (3), (1999) 183-200.

[49] C. D. Pierce, P. Moin, Progress-variable approach for large-eddy simulation of non-premixed turbulent combustion, J. Fluid Mech. 504, (2004) 73-97.

[50] M. Ihme, H. Pitsch, Modeling of radiation and nitric oxide formation in turbulent nonpremixed flames using a flamelet/progress variable formulation, Phys. Fluids 20 (5).

[51] F. S. Lien, H. Liu, E. Chui, C. J. McCartney, Development of an analytical $\beta$-function pdf integration algorithm for simulation of non-premixed turbulent combustion, Flow Turbul. Combust. 83 (2), (2009) 205-226.

[52] N. Peters, Turbulent Combustion, Cambridge University Press (2000).

[53] H. Pitsch, Unsteady flamelet modeling of differential diffusion in turbulent jet diffusion flame, Combust. Flame 123(3), (2000) 358-374.

[54] L. Tesse, F. Dupoirieux, J. Taine, Monte Carlo modeling of radiative transfer in a turbulent sooty flame, Int. J. Heat Mass Transfer 47 (3), (2004) $555-572$.

[55] J. L. Consalvi and F. Nmira, Effects of soot absorption coefficient-Planck function correlation on radiative heat transfer in oxygen-enriched propane turbulent diffusion flame, J. Quant. Spectrosc. Radiat. Transf. 172, (2016) $50-57$.

[56] R. S. Barlow, A. N. Karpetis, J. H. Frank, J. Y. Chen, Scalar profiles and no formation in laminar opposed-flow partially premixed methane/air flames, Combust. Flame 127 (3), (2001) 2102-2118.

[57] M. F. Modest, Radiative Heat Transfer (Third Edition), Academic Press, Boston, 2013, pp. 387-439.

[58] K. C. Smyth, C. R. Shaddix, The elusive history of $\mathrm{m}=1.57-0.56$ i for the refractive index of soot, Combust. Flame 107 (3), (1996) 314-320. 
[59] J. Zhang, C. R. Shaddix, R. W. Schefer, Design of "model-friendly" turbulent non-premixed jet burners for $\mathrm{C} 2+$ hydrocarbon fuels., Rev. Sci. Instrum. 82 (7), (2011) 074101.

[60] Y. Wang, A. Raj, S. H. Chung, A PAH growth mechanism and synergistic effect on PAH formation in counterflow diffusion flames, Combust. Flame 160 (9), (2013) $1667-1676$.

[61] B. Franzelli, B. Fiorina, N. Darabiha, A tabulated chemistry method for spray combustion, Proc. Combust. Inst. 34 (1), (2013) 1659-1666.

[62] T. Schonfeld, Steady and unsteady flow simulations using the hybrid flow solver AVBP, AIAA Journal 37 (11), (1999) 1378-1385.

[63] O. Colin, M. Rudgyard, Development of high-order Taylor-Galerkin schemes for LES, J. Comput. Phys. 162 (2), (2000) 338-371.

[64] T. J. Poinsot, S. K. Lele, Boundary conditions for direct simulations of compressible viscous flows, J. Comput. Phys. 101 (1), (1992) 104-129.

[65] R. Vicquelin, B. Fiorina, S. Payet, N. Darabiha, O. Gicquel, Coupling tabulated chemistry with compressible CFD solvers, Proc. Combust. Inst. 33 (1), (2011) 1481-1488

[66] S. P. Kearney, D. R. Guildenbecher, C. Winters, P. A. Farias, T. W. Grasser, J. C. Hewson, Temperature, Oxygen, and Soot-Volume-Fraction Measurements in a Turbulent C2H4-Fueled Jet Flame, 2015.

[67] C.R. Shaddix, J. Zhang, R.W. Schefer, J. Doom, J.C. Oefelein, S. Kook, L.M. Pickett, H. Wang Understanding and Predicting Soot Generation in Turbulent Non-Premixed Jet Flames, Sandia Report SAND2010-7178, 2010.

[68] International Sooting Flame Workshop website, http://www.adelaide.edu.au/cet/isfworkshop/data-sets/. 
[69] H. El-Asrag, T. Lu, C.K. Law, S. Menon Simulation of soot formation in turbulent premixed flames, Combust. Flame 150 (1), (2007) 108-126.

[70] A. Kronenburg, R. W. Bilger, J. H. Kent, Modeling soot formation in turbulent methane-air jet diffusion flames, Combust. Flame 121 (1), (2000) $24-40$.

[71] Yunardi, R. M. Woolley, M. Fairweather Conditional moment closure prediction of soot formation in turbulent, nonpremixed ethylene flames, Combust. Flame 152 (3), (2008) 360-376.

[72] H. Lalit, J.P. Gore, H. Wang, Assessment of Differential Diffusion Effects on Soot Evolution in Piloted Non-premixed Turbulent Flames, 55th AIAA Aerospace Sciences Meeting, (2017) 1-15.

\section{Appendices}

\section{Appendix A. Soot sectional model source terms}

In this appendix, the detailed formulation of the soot sectional model source terms are detailed.

\section{Nucleation}

Coalescence of two dimers is considered for the formation of the smallest soot nuclei [16, 36] through the Smoluchowski equation [37]. The source term for nucleation is:

$$
\dot{q}_{\mathrm{nu}, i}=v_{\mathrm{d}} \beta_{\mathrm{d}} N_{\mathrm{d}}^{2} \delta_{i 1} / \rho
$$

where $\beta_{\mathrm{d}}$ corresponds to the collision frequency of dimers occuring in a free molecular regime:

$$
\beta_{\mathrm{d}}=\epsilon_{\mathrm{nu}}\left(\frac{3}{4 \pi}\right)^{1 / 6} \sqrt{\frac{6 k_{b} T}{\rho_{s}}} 4 \sqrt{2} v_{\mathrm{d}}^{1 / 6}
$$

where $\epsilon_{\mathrm{nu}}=2.5$ is an amplification factor due to Van der Waals interactions [31]. The Kronecker delta factor $\delta_{i 1}$ in Eq. (A.1) enables the nucleation source 
term to be only considered in the first section of the particle size distribution.

For the LES formalism presented in Sec. 3 and in order to highlight their dependence on the gaseous and solid characteristics, it is convenient to rewrite all the source terms as a product of two contributions. For nucleation, it writes:

$$
\dot{q}_{\mathrm{nu}, 1}=\dot{q}_{\mathrm{nu}, 1}^{\mathrm{gas}} \dot{q}_{\mathrm{nu}, 1}^{\mathrm{solid}},
$$

where:

$$
\begin{aligned}
& \dot{q}_{\mathrm{nu}, 1}^{\mathrm{gas}}=\epsilon_{\mathrm{nu}} \frac{\sqrt{T}}{\rho}\left(\frac{3}{4 \pi}\right)^{1 / 6} \sqrt{\frac{6 k_{b}}{\rho_{s}}} 4 \sqrt{2} v_{\mathrm{d}}^{7 / 6} N_{\mathrm{d}}^{2} \\
& \dot{q}_{\mathrm{nu}, 1}^{\text {solid }}=1
\end{aligned}
$$

\section{Condensation}

It is considered as the coalescence of a dimer at a soot particle surface. The condensation source term of a section $i$ is expressed as:

$$
\dot{q}_{\mathrm{cond}, i}=\dot{q}_{\mathrm{cond}, i}^{\mathrm{gas} \rightarrow i}+\dot{q}_{\mathrm{cond}, i}^{i-1 \rightarrow i}-\dot{q}_{\mathrm{cond}, i}^{i \rightarrow i+1}
$$

where $\dot{q}_{\mathrm{cond}, i}^{\text {gas } \rightarrow i}, \dot{q}_{\text {cond, }+}^{i-1 \rightarrow i}$ and $\dot{q}_{\text {cond,-- }}^{i \rightarrow i+1}$ respectively correspond to the amount of mass of dimer particles that will condensate on particles of section $i$, to the amount of particle mass that will enter into section $i$ due to condensation with particles of section $i-1$, and to the amount of particle mass that will move from section $i$ to section $i+1$ due to condensation. They are respectively evaluated as:

$$
\begin{aligned}
& \dot{q}_{\text {cond }, i}^{\text {gas } \rightarrow i}=\frac{N_{\mathrm{d}}}{\rho} v_{\mathrm{d}} \int_{v_{i}^{\min }}^{v_{i}^{\max }-v_{\mathrm{d}}} \beta_{\mathrm{d}, i} n(w) d w \\
& \dot{q}_{\text {cond }, i}^{i-1 \rightarrow i}=\frac{N_{\mathrm{d}}}{\rho} \int_{v_{i-1}^{\max }-v_{\mathrm{d}}}^{v_{i-1}^{\max }} \beta_{\mathrm{d}, i-1} n(w)\left(w+v_{\mathrm{d}}\right) d w \\
& \dot{q}_{\text {cond }, i}^{i \rightarrow i+1}=\frac{N_{\mathrm{d}}}{\rho} \int_{v_{i}^{\max }-v_{\mathrm{d}}}^{v_{i}^{\max }} \beta_{\mathrm{d}, i} n(w) w d w
\end{aligned}
$$

where the collisional frequency of a dimer with a soot particle has been consid- 
ered constant by section and evaluated at $v_{i}^{\text {mean }}$ :

$$
\begin{aligned}
\beta_{\mathrm{d}, i}= & \epsilon_{\text {cond }}\left(\frac{3}{4 \pi}\right)^{1 / 6} \sqrt{\frac{6 k_{b} T}{\rho_{s}}} \\
& \sqrt{\frac{1}{v_{\mathrm{d}}}+\frac{1}{v_{i}^{\text {mean }}}}\left(v_{\mathrm{d}}^{1 / 3}+\left(\frac{\pi}{6}\right)^{1 / 3} d_{c, i}\right)^{2}
\end{aligned}
$$

where $\epsilon_{\text {cond }}=1.3$ is an amplification factor due to Van der Waals interactions [31]. $\quad d_{c, i}$ corresponds to the collisional diameter of the section $i$ considered constant and evaluated as a function of $n_{p, i}, d_{p, i}$ and the fractal dimension $D_{f}$ of particles (considered equal to 1.8):

$$
d_{c, i}=d_{p, i} n_{p, i}^{1 / D_{f}}
$$

As for nucleation, the gaseous and the solid contributions for the condensation source term have to be separated as:

$$
\dot{q}_{\mathrm{cond}, i}=\dot{q}_{\mathrm{cond}, i}^{\mathrm{gas}} \dot{q}_{\mathrm{cond}, i}^{\mathrm{solid}}
$$

with $\dot{q}_{\mathrm{cond}, i}^{\text {gas }}=\sqrt{T} N_{d} v_{d} / \rho$. Combining Eqs. (A.5) and (A.9), $\dot{q}_{\mathrm{cond}, i}^{\text {solid }}$ is expressed as:

$$
\dot{q}_{\mathrm{cond}, i}^{\mathrm{solid}}=\dot{q}_{\mathrm{cond}, i}^{\mathrm{gas} \rightarrow i, \text { solid }}+\dot{q}_{\mathrm{cond}, i}^{i-1 \rightarrow i, \text { solid }}-\dot{q}_{\mathrm{cond}, i}^{i \rightarrow i, \text { solid }}
$$

with:

$$
\begin{aligned}
& \dot{q}_{\mathrm{cond}, i}^{\mathrm{gas} \rightarrow i, \mathrm{solid}}=\dot{q}_{\mathrm{cond}, i}^{\mathrm{gas} \rightarrow i} / \dot{q}_{\mathrm{cond}, i}^{\mathrm{gas}} \\
& \dot{q}_{\mathrm{cond}, i}^{i-1 \rightarrow \text {, solid }}=\dot{q}_{\mathrm{cond}, i}^{i-1 \rightarrow i} / \dot{q}_{\mathrm{cond}, i}^{\mathrm{gas}} \\
& \dot{q}_{\mathrm{cond}, i}^{i \rightarrow i+1, \text { solid }}=\dot{q}_{\mathrm{cond}, i}^{i \rightarrow i+1} / \dot{q}_{\mathrm{cond}, i}^{\mathrm{gas}} .
\end{aligned}
$$

Doing so, $\dot{q}_{\text {cond }, i}^{\text {gas } \rightarrow i \text { solid }}, \dot{q}_{\text {cond }, i}^{i-1 \rightarrow i \text {,solid }}$ and $\dot{q}_{\text {cond }, i}^{i \rightarrow i+1, \text { solid }}$ only depend on the gaseous phase through $v_{d}$. As a first approximation for the computation of the filtered quantities presented in Sec. $3, \dot{q}_{\mathrm{cond}, i}^{\text {gas } \rightarrow i \text { solid }}, \dot{q}_{\text {cond }, i}^{i-1 \rightarrow i \text {,solid }}$ and $\dot{q}_{\text {cond }, i}^{i \rightarrow i+1, \text { solid }}$ will be computed considering $v_{d}$ equal to $\widehat{v_{d}}$, defined by simplification of Eq. ?? as equal to:

$$
\widehat{v_{d}}=2 \cdot \frac{\sum_{i=1}^{N_{\mathrm{PAH}}} \widetilde{\dot{Q}_{\mathrm{PAH}_{i}}^{\mathrm{d}}}}{\sum_{i=1}^{N_{\mathrm{PAH}}} \widehat{\dot{Q}_{\mathrm{PAH}_{i}}^{\mathrm{d}}} /\left(2 v_{\mathrm{PAH}_{i}}\right)} .
$$




\section{Surface growth and oxidation}

Soot particle surface growth and oxidation occur at its surface. The HACARC mechanism has been employed in the present work [38, 39], and updated for the oxidation reactions by $\mathrm{OH}$ based on recent experimental results [40, 41].

As for condensation and keeping the same notations of Eq. (A.5), the corresponding source terms for a section $i$ write as:

$$
\begin{aligned}
& \dot{q}_{\mathrm{sg}, i}=\dot{q}_{\mathrm{sg}, i}^{\mathrm{gas} \rightarrow i}+\dot{q}_{\mathrm{sg}, i}^{i-1 \rightarrow i}-\dot{q}_{\mathrm{sg}, i}^{i \rightarrow i+1} \\
& \dot{q}_{\mathrm{ox}, i}=-\dot{q}_{\mathrm{ox}, i}^{i \rightarrow \mathrm{gas}}+\dot{q}_{\mathrm{ox}, i}^{i+1 \rightarrow i}-\dot{q}_{\mathrm{ox}, i}^{i \rightarrow i-1}
\end{aligned}
$$

with:

$$
\begin{aligned}
& \dot{q}_{\mathrm{sg}, i}^{\mathrm{gas} \rightarrow i}=\alpha \frac{K_{\mathrm{sg}}}{\rho} v_{C_{2}} \int_{v_{i}^{\min }}^{v_{i}^{\max }-v_{C_{2}}}\left(\frac{w}{v_{C_{2}}}\right)^{\frac{\theta_{i}}{3}} n(w) d w \\
& \dot{q}_{\mathrm{sg}, i}^{i-1 \rightarrow i}=\alpha \frac{K_{\mathrm{sg}}}{\rho} \int_{v_{i-1}^{\max }-v_{C_{2}}}^{v_{i-1}^{\max }}\left(\frac{w}{v_{C_{2}}}\right)^{\frac{\theta_{i-1}}{3}} n(w)\left(w+v_{C_{2}}\right) d w \\
& \dot{q}_{\mathrm{sg}, i}^{i \rightarrow i+1}=\alpha \frac{K_{\mathrm{sg}}}{\rho} \int_{v_{i}^{\max }-v_{C_{2}}^{\max }}^{v^{\max }}\left(\frac{w}{v_{C_{2}}}\right)^{\frac{\theta_{i}}{3}} n(w) w d w
\end{aligned}
$$

and:

$$
\begin{aligned}
& \dot{q}_{\mathrm{ox}, i}^{i \rightarrow \operatorname{gas}}=\alpha v_{C_{2}} \frac{K_{\mathrm{ox}}}{\rho} \int_{v_{i}^{\min }+v_{C_{2}}}^{v_{i}^{\max }}\left(\frac{w}{v_{C_{2}}}\right)^{\frac{\theta_{i}}{3}} n(w) d w \\
& \dot{q}_{\mathrm{ox}, i}^{i+1 \rightarrow i}=\alpha \frac{K_{\mathrm{ox}}}{\rho} \int_{v_{i+1}^{\min }}^{v_{i+1}^{\min }+v_{C_{2}}}\left(\frac{w}{v_{C_{2}}}\right)^{\frac{\theta_{i-1}}{3}} n(w)\left(w-v_{C_{2}}\right) d w \\
& \dot{q}_{\mathrm{ox}, i}^{i \rightarrow i-1}=\alpha \frac{K_{\mathrm{ox}}}{\rho} \int_{v_{i}^{\min }}^{v_{i}^{\min }+v_{C_{2}}}\left(\frac{w}{v_{C_{2}}}\right)^{\frac{\theta_{i}}{3}} n(w) w d w
\end{aligned}
$$

where $\alpha$ is the proportion of sites which are active at the surface of a particle (taken equal to 1 here). $K_{\mathrm{sg}}$ and $K_{\mathrm{ox}}$ are reaction constants obtained with the HACA-RC mechanism. In Eqs. (A.13) and (A.14), only $k_{\mathrm{sg}}=K_{\mathrm{sg}} / \rho, k_{o x}=$ $K_{\mathrm{ox}} / \rho$ depend on the gaseous phase. Then, as for nucleation and condensation, the source terms for surface growth and oxidation can be written as:

$$
\begin{aligned}
& \dot{q}_{\mathrm{sg}, i}=\dot{q}_{\mathrm{sg}, i}^{\mathrm{gas}} \dot{q}_{\mathrm{sg}, i}^{\mathrm{solid}} \\
& \dot{q}_{\mathrm{ox}, i}=\dot{q}_{\mathrm{ox}, i}^{\mathrm{gas}} \dot{q}_{\mathrm{ox}, i}^{\mathrm{solid}}
\end{aligned}
$$


with $\dot{q}_{\mathrm{sg}, i}^{\mathrm{gas}}=k_{\mathrm{sg}}, \dot{q}_{\mathrm{ox}, i}^{\mathrm{gas}}=k_{\mathrm{ox}}$. From Eq. (A.12), $\dot{q}_{\mathrm{sg}, i}^{\mathrm{solid}}$ and $\dot{q}_{\mathrm{ox}, i}^{\text {solid }}$ are expressed as:

$$
\begin{aligned}
& \dot{q}_{\mathrm{sg}, i}^{\mathrm{solid}}=\dot{q}_{\mathrm{sg}, i}^{\mathrm{gas} \rightarrow i, \mathrm{solid}}+\dot{q}_{\mathrm{sg}, i}^{i-1 \rightarrow i, \text { solid }}-\dot{q}_{\mathrm{sg}, i}^{i \rightarrow i+1, \text { solid }} \\
& \dot{q}_{\mathrm{ox}, i}^{\mathrm{solid}}=-\dot{q}_{\mathrm{ox}, i}^{i \rightarrow \text { gas,solid }}+\dot{q}_{\mathrm{ox}, i}^{i+1 \rightarrow i, \text { solid }}-\dot{q}_{\mathrm{ox}, i}^{i \rightarrow i-1, \text { solid }}
\end{aligned}
$$

with:

$$
\begin{array}{ll}
\dot{q}_{\mathrm{sg}, i}^{\mathrm{gas} \rightarrow i, \mathrm{solid}}=\dot{q}_{\mathrm{sg}, i}^{\mathrm{gas} \rightarrow i} / \dot{q}_{\mathrm{sg}, i}^{\mathrm{gas}}, & \dot{q}_{\mathrm{Ox}, i}^{i \rightarrow \mathrm{gas}, \mathrm{solid}}=\dot{q}_{\mathrm{Ox}, i}^{i \rightarrow \mathrm{gas}} / \dot{q}_{\mathrm{Ox}, i}^{\mathrm{gas}}, \\
\dot{q}_{\mathrm{sg}, i}^{i-1 \rightarrow i, \mathrm{solid}}=\dot{q}_{\mathrm{sg}, i}^{i-1 \rightarrow i} / \dot{q}_{\mathrm{sg}, i}^{\mathrm{gas}}, & \dot{q}_{\mathrm{Ox}, i}^{i+1 \rightarrow i, \mathrm{solid}}=\dot{q}_{\mathrm{Ox}, i}^{i+1 \rightarrow i} / \dot{q}_{\mathrm{Ox}, i}^{\mathrm{gas}}, \\
\dot{q}_{\mathrm{sg}, i}^{i \rightarrow i+1, \text { solid }}=\dot{q}_{\mathrm{sg}, i}^{i \rightarrow i+1} / \dot{q}_{\mathrm{sg}, i}^{\mathrm{gas}}, & \dot{q}_{\mathrm{ox}, i}^{i \rightarrow i-1, \mathrm{solid}}=\dot{q}_{\mathrm{ox}, i}^{i \rightarrow i-1} / \dot{q}_{\mathrm{Ox}, i}^{\mathrm{gas}},
\end{array}
$$

\section{Coagulation}

The coagulation corresponds to the collision of two solid particles resulting in a bigger soot particle. When particles from a section $j$ and a section $k$ collide, the resultant particle has a volume comprised in the interval $\left[v_{\min , j}+\right.$ $\left.v_{\min , k} ; v_{\max , j}+v_{\max , k}\right]$. Let us note by $\dot{N}_{\text {coag }}^{j, k \rightarrow i}$ the particle number rate of particles received by the section $i$ from the collision of particles from sections $j$ and $k$, and by $\dot{N}_{i j}^{\text {out }}$ the particle number rate of particles from section $i$ which collide with particles of another section $j$ :

$$
\begin{aligned}
& \dot{N}_{\text {coag }}^{j, k \rightarrow i}=\iint_{v+w \in\left[v_{i}^{\min }, v_{i}^{\max }\right]} \beta_{j, k} n_{j}(v) n_{k}(w) d v d w \\
& \dot{N}_{i j}^{\text {out }}=\int_{v_{i}^{\min }}^{v_{i}^{\max }} \int_{v_{j}^{\min }}^{v_{j}^{\max }} \beta_{i, j} n_{i}(v) n_{j}(w) d v d w
\end{aligned}
$$

It is important to note that $\dot{N}_{\text {coag }}^{j, k \rightarrow i}$ is non-zero only if it exists a particle of volume $v \in\left[v_{j}^{\min }, v_{j}^{\max }\right]$ and a particle of volume $w \in\left[v_{k}^{\min }, v_{k}^{\max }\right]$ respecting $v+w \in\left[v_{i}^{\min }, v_{i}^{\max }\right]$.

The global coagulation source term then writes:

$$
\dot{q}_{\text {coag }, i}=\frac{1}{\rho} \int_{v_{\min , i}}^{v_{\max , i}}\left(\sum_{j, k=1}^{i} \dot{N}_{\text {coag }}^{j, k \rightarrow i}-\sum_{j=1}^{N_{\text {sect }}} \dot{N}_{i j}^{\text {out }}\right) v d v
$$

In these equations, the collision frequency $\beta_{i, j}$ between a particle of section $i$ and a particle of section $j$ is evaluated at $v_{i}^{\text {mean }}$ and $v_{j}^{\text {mean }}$. It is the only term depending on the gaseous phase. Here, a transition regime between the free 
molecular regime (superscript ${ }^{\mathrm{fm}}$ ) and the continuum regime (superscript ${ }^{\mathrm{c}}$ ) has been chosen for the description of collisions. For the collisions between a particle of section $i$ and a particle of section $j, \beta_{i, j}$ is then expressed as:

$$
\beta_{i, j}=\frac{\beta_{i, j}^{\mathrm{fm}} \beta_{i, j}^{\mathrm{c}}}{\beta_{i, j}^{\mathrm{fm}}+\beta_{i, j}^{\mathrm{c}}} \approx \min \left(\beta_{i, j}^{\mathrm{fm}}, \beta_{i, j}^{\mathrm{c}}\right)
$$

with:

$$
\begin{aligned}
\beta_{i, j}^{\mathrm{fm}}= & \epsilon_{\mathrm{coag}}\left(\frac{3}{4 \pi}\right)^{1 / 6} \\
& \sqrt{\frac{6 k_{b} T}{\rho_{s}}} \sqrt{\frac{1}{v_{i}^{\text {mean }}}+\frac{1}{v_{j}^{\text {mean }}}}\left(d_{c, i}+d_{c, j}\right)^{2} \\
\beta_{i, j}^{\mathrm{c}}= & \frac{2 k_{b} T}{3 \mu}\left(d_{c, i}+d_{c, j}\right)\left(\frac{\mathrm{Cu}_{i}}{d_{c, i}}+\frac{\mathrm{Cu}_{j}}{d_{c, j}}\right)
\end{aligned}
$$

where $\epsilon_{\text {coag }}=2.2$ is an amplification factor due to Van der Waals interactions [31], $\mu$ is the gas dynamic viscosity, and $\mathrm{Cu}_{j}$ is the Cunningham corrective coefficient for a particle of section $j$ [32]:

$$
\mathrm{Cu}_{j}=1+1.257 \mathrm{Kn}_{j}=1+1.257 \frac{2 \lambda_{\text {gas }}}{d_{c, j}}
$$

where $\mathrm{Kn}_{j}$ and $d_{c, j}$ are the Knudsen number and collisional diameter of a particle of size $j$, and $\lambda_{\text {gas }}$ is the mean free path of the gaseous phase, expressed by:

$$
\lambda_{\text {gas }}=\frac{R T}{\sqrt{2} \pi d_{\text {gas }}^{2} N_{A} P}
$$

where $R, T, d_{\text {gas }}=0.2 \mathrm{~nm}, N_{A}$ and $P$ correspond respectively to the ideal gas constant, the temperature, the diameter of a typical gas particle, the Avogadro number and the pressure.

Then, equations (A.21) can be written as:

$$
\begin{aligned}
& \beta_{i, j}^{\mathrm{fm}}=\beta_{i, j}^{\mathrm{fm}, \text { gas }} \beta_{i, j}^{\mathrm{fm}, \text { solid }} \\
& \beta_{i, j}^{\mathrm{c}}=\beta_{i, j}^{\mathrm{c} 1, \text { gas }} \beta_{i, j}^{\mathrm{c} 1, \text { solid }}+\beta_{i, j}^{\mathrm{c} 2, \text { gas }} \beta_{i, j}^{\mathrm{c} 2 \text {,solid }}
\end{aligned}
$$


where:

$$
\begin{aligned}
\beta_{i, j}^{\mathrm{fm}, \text { gas }} & =\sqrt{T} \\
\beta_{i, j}^{\mathrm{fm}, \text { solid }} & =\epsilon_{\text {coag }}\left(\frac{3}{4 \pi}\right)^{1 / 6} \sqrt{\frac{6 k_{b}}{\rho_{s}}} \\
& \sqrt{\frac{1}{v_{i}^{\text {mean }}}+\frac{1}{v_{j}^{\text {mean }}}\left(d_{c, i}+d_{c, j}\right)^{2}} \\
\beta_{i, j}^{\mathrm{c} 1 \text { gas }}= & \frac{T}{\mu} \\
\beta_{i, j}^{\mathrm{c} 1, \text { solid }}= & \frac{2 k_{b}}{3}\left(d_{c, i}+d_{c, j}\right)\left(\frac{1}{d_{c, i}}+\frac{1}{d_{c, j}}\right) \\
\beta_{i, j}^{\mathrm{c} 2 \text {,gas }}= & \frac{T \lambda_{\text {gas }}}{\mu} \\
\beta_{i, j}^{\mathrm{c} 2 \text {,solid }}= & \frac{5.028 \cdot k_{b}}{3}\left(d_{c, i}+d_{c, j}\right)\left(\frac{1}{d_{c, i}^{2}}+\frac{1}{d_{c, j}^{2}}\right)
\end{aligned}
$$

with $\beta_{v, w}^{\mathrm{fm}, \text { gas }}, \beta_{v, w}^{\mathrm{c} 1 \text {,gas }}$ and $\beta_{v, w}^{\mathrm{c} 2 \text {,gas }}$ depending only on the gaseous phase quantities $(\mu, P$ and $T)$.

The source term $\dot{q}_{\mathrm{coag}, i}$ is finally expressed as:

$$
\dot{q}_{\mathrm{coag}, i}=\dot{q}_{\mathrm{coag}, i}^{\mathrm{fm}}+\dot{q}_{\mathrm{coag}, i}^{\mathrm{c}}=\dot{q}_{\mathrm{coag}, i}^{\mathrm{fm}}+\dot{q}_{\mathrm{coag}, i}^{\mathrm{c} 1}+\dot{q}_{\mathrm{coag}, i}^{\mathrm{c} 2}
$$

with $\dot{q}_{\mathrm{coag}, i}^{\mathrm{fm}}$ the part of the coagulation source term which rely on a free molecular regime and $\dot{q}_{\mathrm{coag}, i}^{\mathrm{c}}$ the part of the coagulation source term which rely on a continuum regime:

$$
\begin{aligned}
& \dot{q}_{\mathrm{coag}, i}^{\mathrm{fm}}=\dot{q}_{\mathrm{coag}, i}^{\mathrm{fm}, \mathrm{gas}} \dot{q}_{\mathrm{coag}, i}^{\mathrm{fm}, \mathrm{soli}} \\
& \dot{q}_{\mathrm{coag}, i}^{\mathrm{c1}}=\dot{q}_{\mathrm{coag}, i}^{\mathrm{c} 1, \mathrm{~g} a s} \dot{q}_{\mathrm{coag}, i}^{\mathrm{c1}, \mathrm{solid}} \\
& \dot{q}_{\mathrm{coag}, i}^{\mathrm{c} 2}=\dot{q}_{\mathrm{coag}, i}^{\mathrm{c} 2, \mathrm{gas}} \dot{q}_{\mathrm{coag}, i}^{\mathrm{c}, \mathrm{soli}}
\end{aligned}
$$

with:

$$
\begin{array}{ll}
\dot{q}_{\mathrm{coag}, i}^{\mathrm{fm}, \mathrm{gas}}=\frac{\sqrt{T}}{\rho}, & \dot{q}_{\mathrm{coag}, i}^{\mathrm{fm}, \mathrm{solid}}=\dot{q}_{\mathrm{coag}, i}^{\mathrm{fm}} / \dot{q}_{\mathrm{coag}, i}^{\mathrm{fm}, \mathrm{gas}} \\
\dot{q}_{\mathrm{coag}, i}^{\mathrm{c} 1, \mathrm{gas}}=\frac{T}{\mu \rho}, & \dot{q}_{\mathrm{coag}, i}^{\mathrm{c} 1, \mathrm{solid}}=\dot{q}_{\mathrm{coag}, i}^{\mathrm{c} 1} / \dot{q}_{\mathrm{coag}, i}^{\mathrm{c} 1, \mathrm{gas}} \\
\dot{q}_{\mathrm{coag}, i}^{\mathrm{c} 2, \mathrm{gas}}=\frac{T \lambda_{\mathrm{gas}}}{\mu \rho}, & \dot{q}_{\mathrm{coag}, i}^{\mathrm{c} 2, \mathrm{solid}}=\dot{q}_{\mathrm{coag}, i}^{\mathrm{c} 2} / \dot{q}_{\mathrm{coag}, i}^{\mathrm{c} 2, \mathrm{gas}}
\end{array}
$$

